\title{
Electronically competing phases and their magnetic field dependence in electron-doped nonsuperconducting and superconducting $\mathrm{Pr}_{0.88} \mathrm{LaCe}_{0.12} \mathrm{CuO}_{4 \pm \delta}$
}

\author{
H. J. Kang \\ Department of Physics and Astronomy, The University of Tennessee, Knoxville, Tennessee 37996-1200, USA \\ Pengcheng Da: \\ Department of Physics and Astronomy, The University of Tennessee, Knoxville, Tennessee 37996-1200, USA and \\ Condensed Matter Sciences Division, Oak Ridge National Laboratory, Oak Ridge, Tennessee 37831-6393, USA \\ H. A. Mook \\ Condensed Matter Sciences Division, Oak Ridge National Laboratory, Oak Ridge, Tennessee 37831-6393, USA \\ D. N. Argyriou and V. Sikolenko \\ Hahn-Meitner-Institut, Glienicker Str 100, Berlin D-14109, Germany
}

J. W. Lynn

NIST Center for Neutron Research, National Institute of Standards and Technology, Gaithersburg, Maryland 20899, USA

Y. Kurita, Seiki Komiya, and Yoichi Ando

Central Research Institute of Electric Power Industry, Komae, Tokyo 201-8511, Japan

(Dated: March 18, 2018)

\begin{abstract}
We present comprehensive neutron scattering studies of nonsuperconducting and superconducting electron-doped $\mathrm{Pr}_{0.88} \mathrm{LaCe}_{0.12} \mathrm{CuO}_{4 \pm \delta}$ (PLCCO). At zero field, the transition from antiferromagnetic (AF) as-grown PLCCO to superconductivity without static antiferromagnetism can be achieved by annealing the sample in pure Ar at different temperatures, which also induces an epitaxial (Pr,La,Ce $)_{2} \mathrm{O}_{3}$ phase as an impurity. When the superconductivity first appears in PLCCO, a quasi-two-dimensional (2D) spin-density-wave (SDW) order is also induced, and both coexist with the residual three-dimensional (3D) AF state. A magnetic field applied along the $[\overline{1}, 1,0]$ direction parallel to the $\mathrm{CuO}_{2}$ plane induces a "spin-flop" transition, where the noncollinear AF spin structure of PLCCO is transformed into a collinear one. The spin-flop transition is continuous in semiconducting PLCCO, but gradually becomes sharp with increasing doping and the appearance of superconductivity. A $c$-axis aligned magnetic field that suppresses the superconductivity also enhances the quasi-2D SDW order at $(0.5,0.5,0)$ for underdoped PLCCO. However, there is no effect on the $3 \mathrm{D} \mathrm{AF}$ order in either superconducting or nonsuperconducting samples. Since the same field along the $[\overline{1}, 1,0]$ direction in the $\mathrm{CuO}_{2}$ plane has no (or little) effect on the superconductivity, $(0.5,0.5,0)$ and $(\mathrm{Pr}, \mathrm{La}, \mathrm{Ce})_{2} \mathrm{O}_{3}$ impurity positions, we conclude that the $c$-axis field-induced effect is intrinsic to PLCCO and arises from the suppression of superconductivity.
\end{abstract}

PACS numbers: 74.72.Jt, 75.25.+z, 75.50.Ee, 61.12.Ld

\section{INTRODUCTION}

All high-transition-temperature (high- $T_{c}$ ) copper oxides (cuprates) have insulating antiferromagnetic (AF), superconducting (SC), and metallic phases [1]. Understanding the nature of these coexisting and competing phases is a key challenge of current research in high- $T_{c}$ superconductivity [2, 3]. While the SC phase in cuprates arises from hole ( $p$-type) or electron ( $n$-type) doping of the insulating AF phase, it is still unclear how the superconductivity is related to the AF phase [2].

In the hole-doped $\mathrm{La}_{2-x} \mathrm{Sr}_{x} \mathrm{CuO}_{4}$ (LSCO) near $x \sim$ $1 / 8$ [4] and $\mathrm{La}_{2} \mathrm{CuO}_{4+y}$ [5], neutron scattering exper-

*Electronic address: daip@ornl.gov iments have revealed the presence of a static incommensurate spin-density-wave (SDW) order in coexistence with the superconductivity. The SDW is quasi-twodimensional (2D) with long-range order in the $\mathrm{CuO}_{2}$ plane and short-range correlations along the $c$-axis [4, 5]. Indexed on a tetragonal unit cell of LSCO, where the AF order occurs at the reciprocal lattice position $(0.5,0.5)$ in the $\mathrm{CuO}_{2}$ plane, the positions of the four incommensurate SDW peaks are $(0.5 \pm \Delta H, 0.5)$ and $(0.5,0.5 \pm \Delta K)$ as shown in Fig. 1b 1]. At optimal doping (highest $T_{c}$ ), the static SDW order in LSCO vanishes and is replaced by the appearance of a spin gap and incommensurate spin fluctuations at energies above the gap [6]. On the other hand, experiments on lightly doped insulating $(x=0.03$, $0.04,0.05)$ and underdoped SC $(x=0.06)$ LSCO have shown that the static SDW order appears in these samples, but intriguingly the four incommensurate peaks in Fig. $1 \mathrm{~b}$ are only observed with the establishment of the 


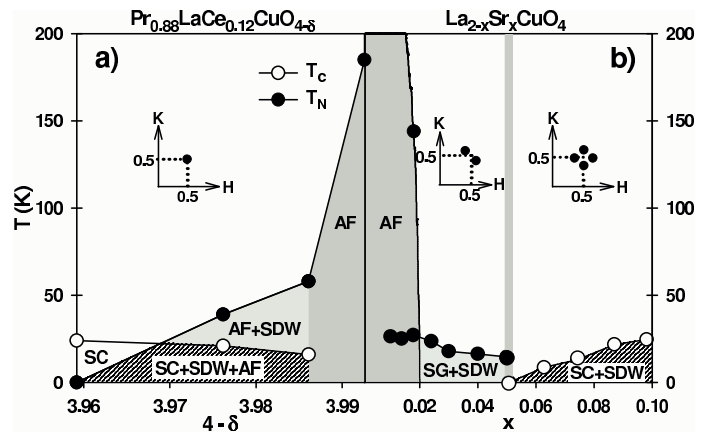

FIG. 1: Comparison of the phase diagrams between hole-doped $\mathrm{La}_{2-x} \mathrm{Sr}_{x} \mathrm{CuO}_{4}$ (LSCO) and electron-doped $\mathrm{Pr}_{0.88} \mathrm{LaCe}_{0.12} \mathrm{CuO}_{4 \pm \delta}$ (PLCCO). a) Properties of the PLCCO samples investigated in this work. Since the exact oxygen concentrations in our samples are unknown, the values of $4-\delta$ in the horizontal axis are obtained using Fig. 8 of Ref. [32] by assuming that PLCCO samples with the same $T_{c}$ 's as those of $\mathrm{Nd}_{1.85} \mathrm{Ce}_{0.15} \mathrm{CuO}_{4-\delta}$ have the same oxygen content. When superconductivity is first established in PLCCO, a commensurate static SDW order also appears. The open and filled circles are data from Ref. [36]. b) Phase diagram of LSCO as a function Sr-doping from neutron scattering results of Ref. [7, 8, 9]. In the spin-glass (SG) phase of LSCO, two static incommensurate SDW peaks at $(0.5+\Delta H, 0.5-\Delta K)$ and $(0.5-\Delta H, 0.5+\Delta K)$ (see inset) are observed for $0.02 \leq x \leq 0.05$. When superconductivity sets in at $x \geq 0.05$, two pairs of incommensurate SDW peaks appear simultaneously at $(0.5 \pm \Delta H, 0.5)$ and $(0.5,0.5 \pm \Delta K)$. The data in b) are from Refs. [7, 8, 9].

bulk superconductivity for the underdoped SC samples $(0.06 \leq x \leq 0.12)$ [7, 8, [9]. Therefore, the insulating-tosuperconducting phase transition induced by increasing hole(Sr)-doping is associated with the appearance of a static quasi-2D SDW modulation.

When a magnetic field is applied along the $c$-axis of the underdoped SC LSCO, it not only suppresses the superconductivity but also enhances the zero-field SDW order 10, 11, 12, 13]. For optimally doped LSCO without static SDW order, a $c$-axis magnetic field induces spin fluctuations below the spin-gap energy and drives the system toward static AF order 14, 15]. Since the same field applied in the $\mathrm{CuO}_{2}$ plane has little effect on superconductivity or the SDW order [16], these results suggest that antiferromagnetism is a competing ground state revealed by the suppression of superconductivity [17, 18, 19, 20, 21, 22, 23].

If suppression of superconductivity in hole-doped LSCO indeed drives the system toward an AF ordered state, it is important to determine the universality of such a feature in other SC copper oxides [24]. Although neutron scattering experiments failed to confirm any enhancement of the static long-range $\mathrm{AF}$ order in hole-doped $\mathrm{YBa}_{2} \mathrm{Cu}_{3} \mathrm{O}_{6.6}$ for a $c$-axis aligned field up to 7-T [25, 26], these measurements may not be conclusive because of the enormous upper critical fields $B_{c 2}$ ( $>45$-T for $c$-axis aligned fields) required to completely
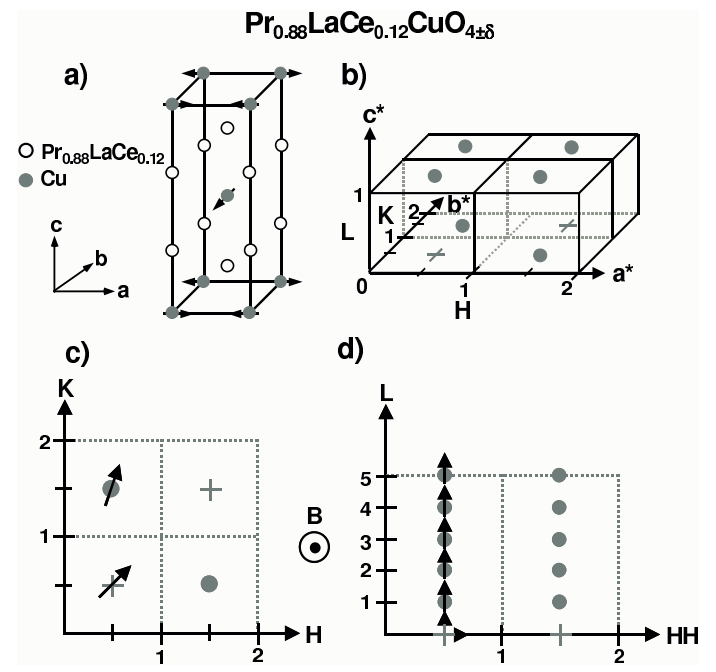

FIG. 2: a) Schematic diagram of the noncollinear spin structure of PLCCO, b) Allowed AF Bragg Peaks from the spin structure of a), c) Reciprocal space probed in the $[H, K, 0]$ scattering plane where the applied field is along the $c$-axis. Solid circles are allowed AF Bragg peak positions in the $[H, K, 0]$ plane. d) Similar experiments in the $[H, H, L]$ zone where the field is along the $[\overline{1}, 1,0]$ direction in the $\mathrm{CuO}_{2}$ plane. Arrows indicate the scan directions in our experiments.

suppress superconductivity. Compared to hole-doped cuprates, electron-doped materials offer a unique opportunity for studying the magnetic field-induced effect for two reasons. First, electron-doped materials generally have upper critical fields less than 15-T [27, 28, 29], a value reachable in neutron scattering experiments. Second, electron-doped materials can be transformed from as-grown nonsuperconducting (NSC) AF insulators to full superconductivity by simply annealing the samples at different temperatures to modify the charge carrier density [30, 31, 32, 33, 34]. This unique property allows the possibility of studying the NSC to SC transition in electron-doped cuprates without the complications of structural and/or chemical disorder induced by chemical substitution in hole-doped materials. Recently, we discovered that when superconductivity first appears in one family of electron-doped superconductors, $\mathrm{Pr}_{0.88} \mathrm{LaCe}_{0.12} \mathrm{CuO}_{4 \pm \delta}$ (PLCCO), a commensurate quasi-2D SDW order is also induced [35], and both coexist with the residual three-dimensional (3D) AF state [36. The optimally doped SC PLCCO, on the other hand, has no static SDW or residual AF order [34, 36], analogous to hole-doped LSCO as depicted in Fig. 1 [6, [7, [8, 9].

For prototypical electron-doped $\mathrm{Nd}_{2-x} \mathrm{Ce}_{x} \mathrm{CuO}_{4}$ (NCCO) [30, 31], previous neutron scattering experiments show a drastic suppression of the static 3D AF order when superconductivity is established [37]. However, the static AF order persists even for NCCO with optimal bulk superconductivity $\left(T_{c}=25 \mathrm{~K}\right) 38$. In the initial neutron scattering experiments on the 
slightly underdoped $\mathrm{SC} \mathrm{Nd}_{1.86} \mathrm{Ce}_{0.14} \mathrm{CuO}_{4}$, Matsuda et al. 39] found that a 10-T c-axis-aligned magnetic field has no effect on the residual AF order in the sample. On the other hand, we discovered that such fields enhance magnetic intensities at $\mathrm{AF}$ ordering positions in an optimally doped $\mathrm{Nd}_{1.85} \mathrm{Ce}_{0.15} \mathrm{CuO}_{4}$ [40, 41]. The induced AF moments scale approximately linearly with the applied field, saturate at $B_{c 2}$, and then decrease for higher fields. These results thus indicate that AF order is a competing ground state to superconductivity in electron-doped $\mathrm{Nd}_{1.85} \mathrm{Ce}_{0.15} \mathrm{CuO}_{4}$ [42].

Although electron-doped NCCO offers a unique opportunity for studying the superconductivity-suppressed ground state of high- $T_{c}$ cuprates, the system has one important complication. The as-grown material is NSC, and has to be oxygen reduced to render the system superconducting. This reducing process has been found to produce a small quantity of the cubic $(\mathrm{Nd}, \mathrm{Ce})_{2} \mathrm{O}_{3}$ as an impurity phase [41, 43, 44, 45, 46]. Generally, a small amount $(0.01 \%$ to $1 \%)$ of randomly distributed impurity would be unobservable, but $(\mathrm{Nd}, \mathrm{Ce})_{2} \mathrm{O}_{3}$ stabilizes as an oriented crystalline lattice in the NCCO matrix because its lattice constant is about $2 \sqrt{2}$ larger than the planner lattice constant of the tetragonal NCCO $(a=3.945$ $\AA$ and $\left.a_{N O}=2 \sqrt{2} a\right)$. This in-plane lattice match with $\mathrm{NCCO}$ means reflections at $(H, K, 0)$ of $\mathrm{NCCO}$ in reciprocal space may also stem from $(\mathrm{Nd}, \mathrm{Ce})_{2} \mathrm{O}_{3}$, thus giving structural impurity peaks that match some of the magnetic peaks [such as $(0.5,0.5,0)]$. In the paramagnetic state of $(\mathrm{Nd}, \mathrm{Ce})_{2} \mathrm{O}_{3}$, a field induces a net magnetization on $\mathrm{Nd}^{3+}$, which can then contaminate the intensity of the cuprate magnetic peak such as $(0.5,0.5,0)$ 41, 43, 44, 45, 46].

There are three ways to resolve this impurity problem and determine the intrinsic properties of electron-doped materials. First, since the impurity and cuprate peaks are not lattice matched along the $c$-axis direction (the lattice constant of impurity is about $10 \%$ smaller than that of NCCO), the impurity peaks occur at positions such as $(0.5,0.5, L) L=2.2,4.4$, and so on [41, 43]. This allows the impurity scattering to be determined separately, and the in-plane scattering can then be corrected by subtracting the impurity contribution to ascertain the NCCO contribution of the in-plane peaks [41, 45. Second, with a $c$-axis-aligned magnetic field and $c$-axis in the scattering plane, the non-zero integer $L$ positions $(L=1,2,3, \cdots)$ can be measured without any possible impurity contribution. We carried out such an experiment using a horizontal field magnet and found that application of a 4-T $c$-axis aligned magnetic field enhances the $(0.5,0.5,3) \mathrm{AF}$ reflection in the optimally doped $\mathrm{SC} \mathrm{Nd}{ }_{1.85} \mathrm{Ce}_{0.15} \mathrm{CuO}_{4}$ [1]. Both procedures show unambiguously that there is a field-induced moment in electron-doped $\mathrm{Nd}_{1.85} \mathrm{Ce}_{0.15} \mathrm{CuO}_{4}$, whose behavior is similar to that observed in the hole-doped systems.

Although our previous experiments 40, 41, 45] have demonstrated the intrinsic nature of the field-induced effect in $\mathrm{SC} \mathrm{Nd}_{1.85} \mathrm{Ce}_{0.15} \mathrm{CuO}_{4}$, it is still interesting to

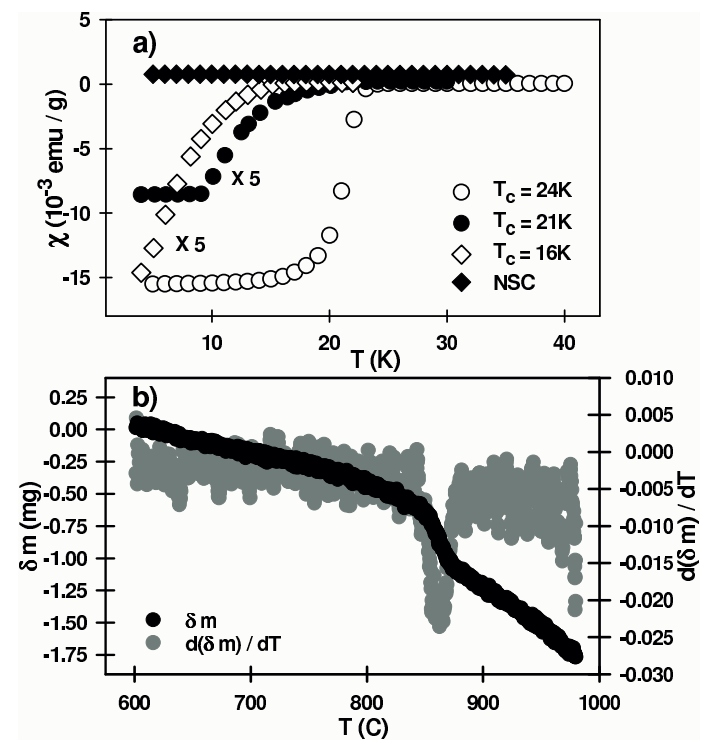

FIG. 3: a) Magnetic susceptibility measurements of $\mathrm{Pr}_{0.88} \mathrm{LaCe}_{0.12} \mathrm{CuO}_{4 \pm \delta}$ single crystals under a small (510 Gauss) field in the $\mathrm{CuO}_{2}$ plane. b) Temperature dependence of weight change and its derivative of a $\operatorname{Pr}_{1.18} \mathrm{La}_{0.7} \mathrm{Ce}_{0.12} \mathrm{CuO}_{4-\delta}$ in the annealing process.

perform field-induced experiments on samples free of $\mathrm{Nd}$ because application of a magnetic field is known to induce a large moment on rare-earth $\mathrm{Nd}^{3+}$ spins that may conceal the intrinsic field dependence of the $\mathrm{Cu}^{2+}$ moments. Electron-doped $\mathrm{Pr}_{1-x} \mathrm{LaCe}_{x} \mathrm{CuO}_{4 \pm \delta}$, where $\mathrm{Pr}^{3+}$ has a singlet ground state [7] and La is nonmagnetic, is ideal for this purpose. Using single crystals of $\operatorname{Pr}_{1-x} \mathrm{LaCe}_{x} \mathrm{CuO}_{4 \pm \delta}$ with $x=0.11\left(T_{c}=16 \mathrm{~K}\right)$ and $x=0.15\left(T_{c}=26 \mathrm{~K}\right)$, Fujita et al. [48] found that a $c$-axis aligned magnetic field enhances the residual AF order in the $x=0.11$ sample at $(0.5,1.5,0)$ position, but has no effect in the overdoped $x=0.15$ sample. The authors conclude that a $c$-axis field changes both the magnetic intensity and the onset of the Néel ordering temperature $T_{N}$ for the $x=0.11$ sample, with the maximum field effect at $\sim 5-\mathrm{T}[48$.

Our approach to this problem has been slightly different from that of Fujita et al. [48]. Instead of preparing different $\mathrm{SC} \mathrm{Pr}_{1-x} \mathrm{LaCe}_{x} \mathrm{CuO}_{4 \pm \delta}$ samples as a function of Ce-doping, we grew single crystals of PLCCO using a traveling solvent floating zone furnace. As-grown, $\mathrm{PLCCO}$ has a noncollinear $\mathrm{Cu}$ spin structure as shown in Fig. 2a with a small induced $\mathrm{Pr}$ moment due to $\mathrm{Cu}-\mathrm{Pr}$ interaction [49]. When the AF ordered semiconducting PLCCO is transformed into a superconductor by annealing in pure $\mathrm{Ar}$, the $\mathrm{SC}$ transition temperatures $\left(T_{c}\right.$ 's) can be controlled by judicially tuning the annealing temperature [34]. This approach avoids complications of Cesubstitution for different $T_{c}$ materials. For underdoped samples with $T_{c}$ 's below $25 \mathrm{~K}$, superconductivity coexists with both the 3D AF-type order of the undoped material and a commensurate quasi-2D SDW modulation [36]. 
Optimally doped PLCCO has no static SDW or residual AF order.

In this article, we present comprehensive neutron scattering studies of four single crystals of $n$-type PLCCO from a semiconductor with $T_{N}=186 \mathrm{~K}$ to superconductors with $T_{c}=16 \mathrm{~K}, 21 \mathrm{~K}$, and $24 \mathrm{~K}$, respectively (Figs. 1a and 3a). When a magnetic field is applied along the $[\overline{1}, 1,0]$ direction parallel to the $\mathrm{CuO}_{2}$ plane, the noncollinear AF spin structure of PLCCO is transformed into a collinear one through a "spin-flop" transition [49]. Such a spin-flop transition is continuous in semiconducting PLCCO but gradually changes to discontinuous with increasing doping and the appearance of superconductivity. For SC PLCCO, an in-plane field only induces a spin-flop transition but does not change the effective $\mathrm{Cu}^{2+}$ or $\mathrm{Pr}^{3+}$ moments for fields up to 14T. A $c$-axis aligned magnetic field, on the other hand, enhances the quasi-2D SDW order at $(0.5,0.5,0)$ for underdoped PLCCO, but has no effect on the 3D AF order in the NSC and SC samples. Since the same 14-T field along the $[\overline{1}, 1,0]$ direction parallel to the $\mathrm{CuO}_{2}$ plane has no effect on $(0.5,0.5,0)$ and on the lattice Bragg peaks of the $(\mathrm{Pr}, \mathrm{La}, \mathrm{Ce})_{2} \mathrm{O}_{3}$ impurity phase, we conclude that the $c$-axis field-induced effect is intrinsic to PLCCO and arises from the suppression of superconductivity.

The rest of the paper is organized as follows: in Sec. II we describe the details of sample preparation methods and neutron-scattering experimental setup. The main experimental results are summarized in Sec. III, where Sec. III.A reviews the zero-field magnetic properties, Sec. III.B discusses the effects of in-plane magnetic fields on the AF spin structure, Sec. III.C summarizes the magnetic field effect on the NSC PLCCO and impurity $(\mathrm{Pr}, \mathrm{La}, \mathrm{Ce})_{2} \mathrm{O}_{3}$ phase, Sec. III.D presents the comprehensive $c$-axis field effect studies and their temperature dependence for different SC PLCCO samples, and finally Sec. III.E discuss the anisotropy of the field effect by comparing the in-plane and $c$-axis field effect on the $T_{c}=21 \mathrm{~K}$ SC sample. In Sec. IV, we compare our results with previous experiments in $p$ - and $n$-type materials. A brief summary is given in Sec. V.

\section{EXPERIMENTAL DETAILS}

We grew high quality PLCCO single crystals (cylindrical rods weight $0.8-1.5$ grams with mosaicity $<1^{\circ}$ ) using the traveling solvent floating zone technique and annealed the samples in pure Ar at different temperatures to control the SC transition temperatures. The partial substitution of Pr with La was used to stabilize the crystal growth without introducing significant lattice distortions [50]. We obtained three SC PLCCO samples with the onset temperature for bulk superconductivity at $T_{c}=24 \mathrm{~K}, 21 \mathrm{~K}$, and $16 \mathrm{~K}$ from magnetic susceptibility measurements (Fig. 3a). The $T_{c}=24 \mathrm{~K}$, and 21 $\mathrm{K}$ samples are obtained by annealing the as-grown single crystals in pure argon at $970^{\circ} \mathrm{C}$ and $940^{\circ} \mathrm{C}$ for 24 hours,

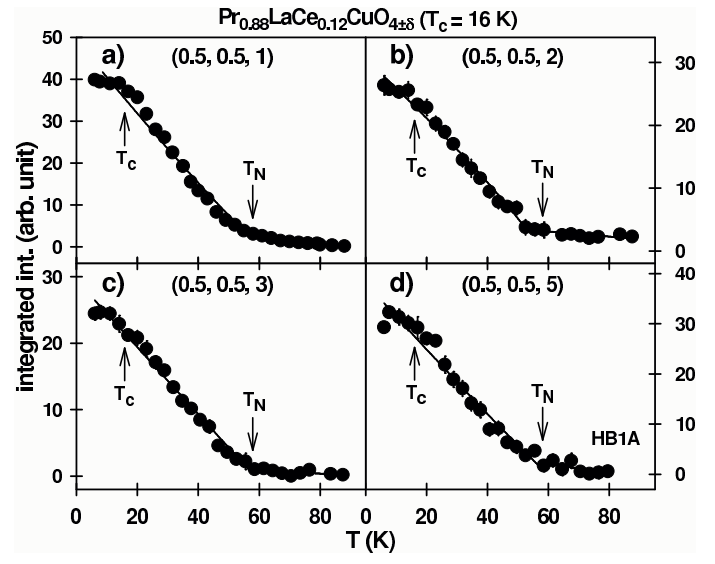

FIG. 4: Temperature dependence of integrated intensities at $(0.5,0.5, L)$ for the $T_{c}=16 \mathrm{~K} \mathrm{PLCCO}$. The solid lines are guides to the eye. The arrows indicate $T_{c}$ and $T_{N}$, respectively.

respectively. To obtain the $T_{c}=16 \mathrm{~K}$ crystal, the asgrown sample was annealed in pure argon at $915^{\circ} \mathrm{C}$ for 1 week. The SC PLCCO of $T_{c}=21 \mathrm{~K}$ and $16 \mathrm{~K}$ are near the phase boundary between AF and SC phases and the $T_{c}=24 \mathrm{~K}$ sample is in the optimally doped regime. We also obtained a NSC sample by annealing one of the SC $T_{c}=16 \mathrm{~K}$ samples in air at $900^{\circ} \mathrm{C}$ for 24 hours [36].

One of the open questions in electron-doped superconductors is the role of the annealing process to superconductivity [30]. In earlier works, it was postulated that as-grown NSC samples have excess oxygen atoms above copper sites (apical oxygen). These extraneous oxygen atoms have been believed to induce a local disordered potential that localizes doped electrons and therefore prohibits superconductivity [51]. When the annealing process removes these defect oxygen atoms at apical sites, the electrons will not be localized and the superconductivity appears. The magnitude of the Hall coefficient in annealed NCCO $(x=0.15)$ decreases dramatically below $T<100 \mathrm{~K}$, very differently from the as-grown sample [51]. This suggests that annealing changes the mobile charge density. To understand what annealing does to our PLCCO samples, we have performed thermogravimetric analysis (TGA) of our samples under different annealing conditions. Figure $3 \mathrm{~b}$ shows the temperature dependence of the weight change for a $\operatorname{Pr}_{1.18} \mathrm{La}_{0.7} \mathrm{Ce}_{0.12} \mathrm{CuO}_{4 \pm \delta}$ sample during the annealing process. The large drop in weight change shows up as a kink in the data and a sharp dip in its derivative; we interpret this kink to be indicative of the point where the oxygen content becomes the closest to stoichiometry, namely $\delta \simeq 0$ 50]. In any case, the TGA data give the most direct evidence that oxygen content of the sample is reduced during the annealing process.

While TGA analysis indicates a decreased oxygen content after the reduction process, it is still unclear whether the apical oxygen is removed 52,53. Indeed, recent Raman and crystal-field infrared transmission results sug- 
gest that instead of removing apical oxygen as originally thought, reduction of optimally doped $\operatorname{Pr}_{2-x} \mathrm{Ce}_{x} \mathrm{CuO}_{4 \pm \delta}$ and NCCO samples actually involves only oxygens in the $\mathrm{CuO}_{2}$ plane [54, 55]. Clearly, the exact oxygen content and their arrangements in the crystal before and after the reduction need to be sorted out by future studies.

Another issue to be noted regarding the PLCCO material is the relevance of the $(\mathrm{Pr}, \mathrm{La}, \mathrm{Ce})_{2} \mathrm{O}_{3}$ impurity phase, which plays the same role in PLCCO as the $(\mathrm{Nd}, \mathrm{Ce})_{2} \mathrm{O}_{3}$ phase in NCCO. To understand the effect of annealing on this impurity phase, we have performed systematic synchrotron X-ray diffraction investigations on as-grown NSC, SC, and re-oxygenated NSC PLCCO samples at the Advanced Photon Source, Argonne National Laboratory. We confirm that the $(\mathrm{Pr}, \mathrm{La}, \mathrm{Ce}){ }_{2} \mathrm{O}_{3}$ impurity phase can be produced by annealing and but it vanishes after the reoxygenation process [33]; such a behavior is rather strange, but is understandable if the host PLCCO crystals are slightly $\mathrm{Cu}$ deficient. To confirm this possibility, inductively-coupled plasma atomic-emission spectroscopy (ICP-AES) analysis was performed on a batch of crystals that are nominally $\operatorname{Pr}_{1.18} \mathrm{La}_{0.7} \mathrm{Ce}_{0.12} \mathrm{CuO}_{4 \pm \delta}$. The reference solution is prepared by diluting commercial standard solutions not by the volume but by the weight, which enables us to calculate the cation concentration of the reference with a high resolution. A piece of the $\operatorname{Pr}_{1.18} \mathrm{La}_{0.7} \mathrm{Ce}_{0.12} \mathrm{CuO}_{4 \pm \delta}$ crystal $(1 \mathrm{mg})$ is dissolved into a $30 \mathrm{~cm}^{3}$ of $\mathrm{HNO}_{3}(1 \mathrm{Mol} /$ Liter $)$. The analyzed cation ratio of the nominally $\operatorname{Pr}_{1.18} \mathrm{La}_{0.7} \mathrm{Ce}_{0.12} \mathrm{CuO}_{4 \pm \delta}$ sample was Pr : La : $\mathrm{Ce}: \mathrm{Cu}=1.20: 0.68: 0.12: 0.97$, with a relative error of less than $1 \%$ for each element. This result shows that $\mathrm{Cu}$ is indeed deficient by about $3 \%$ in the $\operatorname{Pr}_{1.2} \mathrm{La}_{0.7} \mathrm{Ce}_{0.12} \mathrm{CuO}_{4 \pm \delta}$ crystal, which is probably also true for PLCCO. Assuming that as-grown PLCCO samples are slightly $\mathrm{Cu}$ deficient as $\mathrm{Pr}_{1.2} \mathrm{La}_{0.7} \mathrm{Ce}_{0.12} \mathrm{CuO}_{4 \pm \delta}$, the effect of annealing is then to remove oxygen in PLCCO to form the $(\mathrm{Pr}, \mathrm{La}, \mathrm{Ce})_{2} \mathrm{O}_{3}$ impurity and make the remaining crystal structure more perfect for superconductivity. This analysis can potentially explain why the $(\mathrm{Pr}, \mathrm{La}, \mathrm{Ce})_{2} \mathrm{O}_{3}$ impurity phase can be reversibly produced [33].

Our neutron scattering measurements were performed on the HB-1A, HB-1, and HB-3 triple-axis spectrometers at the high-flux-isotope reactor (HFIR), Oak Ridge National Laboratory (ORNL) and on the E4 twoaxis diffractometer and E1 triple-axis spectrometer at the Berlin Neutron Scattering Center, Hahn-MeitnerInstitute (HMI). The field effect experiments on SC samples of $T_{c}=24 \mathrm{~K}, 21 \mathrm{~K}$, and $16 \mathrm{~K}$ and the NSC sample $\left(T_{N}=186 \mathrm{~K}\right)$ were carried out at HFIR using a $7-\mathrm{T}$ vertical field SC magnet 25]. The collimations were $48^{\prime}$ $40^{\prime}$-sample-40'-102' (full-width at half maximum) from the reactor to the detector and the final neutron energies were fixed at either $E_{f}=14.6 \mathrm{meV}$ or $13.5 \mathrm{meV}$. For high magnetic field experiments, we used the VM-1 14.5$\mathrm{T}$ vertical field SC magnet at HMI and a $40^{\prime}-40^{\prime}$-sample$40^{\prime}$ collimation with a fixed neutron final energy of 13.6 meV. A pyrolytic graphite (PG) monochromator and $\mathrm{PG}$

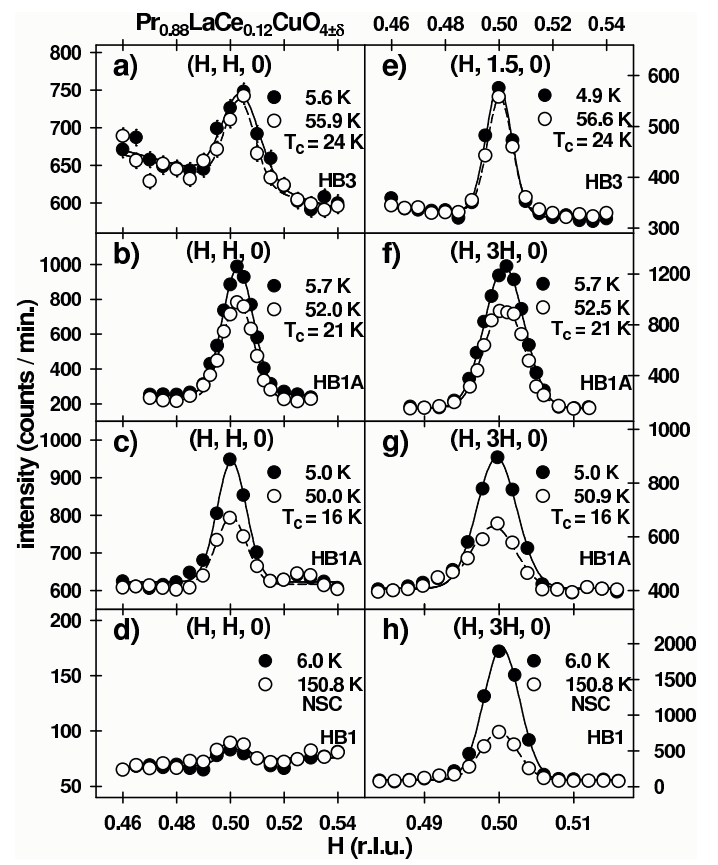

FIG. 5: $\mathbf{Q}$ scans in the $[H, K, 0]$ zone at low and high temperatures for different PLCCO samples. a-d) Scans along the $[H, H, 0]$ direction around $(0.5,0.5,0)$ for $T_{c}=24 \mathrm{~K}, 21 \mathrm{~K}, 16$ $\mathrm{K}$, and NSC PLCCO, e-h) Similar scans along the $[H, 3 H, 0]$ direction around $(0.5,1.5,0)$. This figure is reproduced from Fig. 2 of Ref. [36].

analyzer were used and PG filters were placed in front of the sample to remove higher order contaminations from the incident beam. The samples were clamped on solid aluminum brackets similar to those used in the NCCO experiments [41]. However, a $c$-axis aligned field almost exerts no torque on PLCCO because of the nonmagnetic singlet nature of the Pr ground state.

We use elastic neutron scattering to probe AF order and label the momentum transfer $\mathbf{Q}=\left(q_{x}, q_{y}, q_{z}\right)$ in $\AA^{-1}$ as $(H, K, L)=\left(q_{x} a / 2 \pi, q_{y} b / 2 \pi, q_{z} c / 2 \pi\right)$ in the reciprocal lattice units (r.l.u.) appropriate for the tetragonal unit cell of PLCCO (space group $I 4 / \mathrm{mmm}, a=b=3.98$ and $c=12.27 \AA$ ). Here $a$ and $c$ are in-plane and outof -plane lattice constants, respectively. The crystals were aligned in two different geometries, $[H, K, 0]$ and $[H, H, L]$ scattering planes. The vertical magnetic field was applied along the $c$-axis in the former scattering plane and was applied along the $[\overline{1}, 1,0]$ direction $(\mathbf{B} \| a b$ plane) in the later case. The $[H, H, L]$ geometry was also used to search for quasi-2D SDW order at zero-field using a two-axis energy integrated mode (Fig. 2d), to determine the field-induced effect on the impurity phase, and investigate the field-induced spin-flop transitions. In the $[H, K, 0]$ geometry, we studied the $c$-axis field-induced effect on SC and NSC PLCCO. The anisotropy of the field-induced effect can be determined by comparing the results in these two geometries since the magnetic field along the $c$-axis suppresses the SC much more strongly 


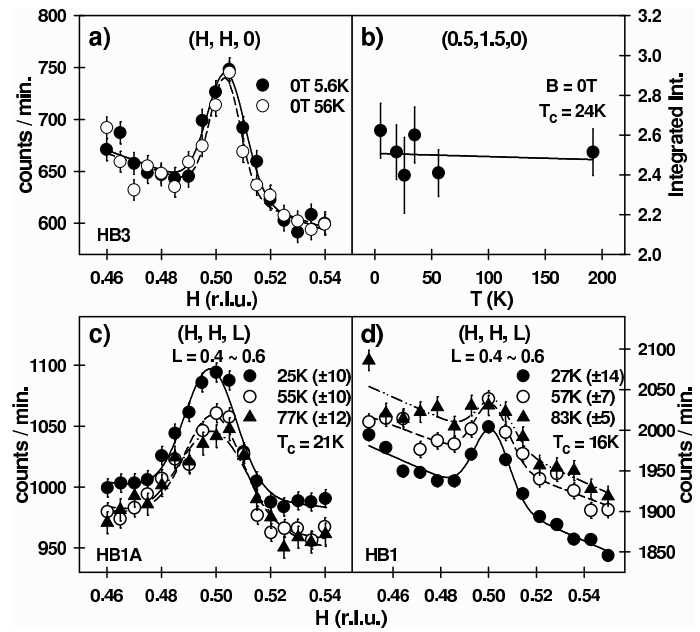

FIG. 6 : a) $[H, H, 0]$ scans around $(0.5,0.5,0)$ at $5.6 \mathrm{~K}$ and 56 $\mathrm{K}$ for the optimally doped $T_{c}=24 \mathrm{~K}$ PLCCO. The observed peak at $(0.5,0.5,0)$ has nonmagnetic origin. b) The temperature dependence of the integrated intensities at $(0.5,1.5,0)$ for the $T_{c}=24 \mathrm{~K}$ PLCCO. There is no evidence for static SDW and/or AF orders in the optimally doped PLCCO. cd) Two-axis mode scans along the $[H, H, 0]$ direction around $(0.5,0.5, L)(0.4<L<0.6)$ at different temperatures for

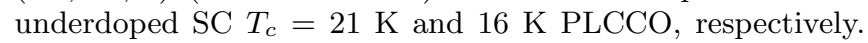
Magnetic scattering clearly decreases with increasing temperature for both compounds.

than that for the same field in the $a b$-plane.

\section{RESULTS}

\section{A. Magnetic properties of superconducting (SC) and nonsuperconducting (NSC) \\ $\mathrm{Pr}_{0.88} \mathrm{LaCe}_{0.12} \mathrm{CuO}_{4 \pm \delta}$ at zero-field}

To determine the influence of a magnetic field on the $\mathrm{AF}$ order of PLCCO, it is helpful to know the magnetic structure of its parent compound. $\mathrm{Pr}_{2} \mathrm{CuO}_{4}$ has a bodycentered tetragonal structure with space group $I 4 / \mathrm{mmm}$ [56]. The spins of $\mathrm{Cu}^{2+}$ ions have AF ordering in the $\mathrm{CuO}_{2}$ plane due to strong in-plane exchange interaction. The orientation of spins in adjacent planes is noncollinear governed by the weak pseudo-dipolar interaction between planes, since exchange field on each copper ion by neighboring planes is canceled due to body-centered tetragonal crystal symmetry $[56]$. The spins at $(0,0,0)$ and $(0.5,0.5,0.5)$ positions are along the $[1,0,0]$ and $[0, \overline{1}, 0]$ directions, respectively (Fig. 2a). In this magnetic structure, the AF Bragg peak at $(0.5,0.5,0)$ is disallowed. The allowed magnetic Bragg peaks are at $(0.5,0.5, L)$ $(L=1,2,3, \cdots)$ and $(0.5,1.5, L)(L=0,1,2,3, \cdots)$ marked as solid dots in Fig. 2b.

We performed neutron diffraction measurements at zero-field on magnetic Bragg peaks $(0.5,0.5, L)$ ( $L=$ $1,2,3,5)$ to determine the magnetic structure of the

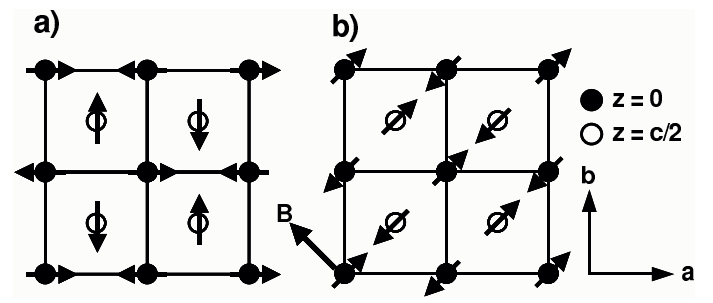

FIG. 7: Magnetic structures of $\mathrm{Cu}^{2+}$ ions in PLCCO. a) Noncollinear structure at zero-field. b) Collinear structure transformed by a magnetic field applied along the $[\overline{1}, 1,0]$ direction.

superconducting $T_{c}=16 \mathrm{~K}$ PLCCO (Fig. 4). In principle, one cannot uniquely determine the spin structure of PLCCO based on magnetic intensities at $(0.5,0.5, L)$ alone. However, since the spin structure of $\mathrm{Pr}_{2-x} \mathrm{Ce}_{x} \mathrm{CuO}_{4}$ at all $\mathrm{Ce}$ doping levels is non-collinear as depicted in Fig. 2a [56], one would expect a similar magnetic structure in PLCCO because substitution of $\mathrm{La}^{3+}$ for $\mathrm{Pr}^{3+}$ will weaken the pseudo-dipolar interactions but not eliminate it. The integrated intensity comparison of $(0.5,0.5, L)$ peaks to the calculated intensities confirms that the superconducting sample has the same noncollinear spin structure as that of the parent $\mathrm{Pr}_{2} \mathrm{CuO}_{4}$.

To estimate the effective $\mathrm{Cu}^{2+}$ and $\mathrm{Pr}^{3+}$ moments, we measured the temperature dependence of $(0.5,0.5, L)$ reflections. By comparing the magnetic structure factor calculations (Table I) with that of the weak nuclear Bragg peak $(1,1,0)$, we calculate an ordered $\mathrm{Cu}^{2+}$ moment of $0.06 \pm 0.01 \mu_{B}$ at $5 \mathrm{~K}$ with negligible induced moment on $\mathrm{Pr}^{3+}$ ions for the $T_{c}=16 \mathrm{~K}$ PLCCO [57]. Since the induced $\mathrm{Pr}^{3+}$ moment contributes positively to the intensity of $(0.5,0.5,1)$ and $(0.5,0.5,2)$, but negatively to $(0.5,0.5,3)$, the temperature dependent measurements of $(0.5,0.5, L)$ should reveal deviations from power law fits when the Pr-induced moment becomes significant [49]. In addition, the $(0.5,0.5,5)$ peak should exhibit only the temperature dependence of the $\mathrm{Cu}^{2+}$ moment as there are essentially no $\mathrm{Pr}^{3+}$ moment contributions to this reflection (Table I). Since all magnetic peaks at $(0.5,0.5, L)$ show a similar temperature dependence as $(0.5,0.5,5)$ (Fig. 4), we conclude that there is negligible Pr-induced moment above $4 \mathrm{~K}$ in the $T_{c}=16 \mathrm{~K}$ PLCCO.

In a recent work, we have systematically investigated how superconductivity develops from the AF ordered insulating state in PLCCO [36]. By carefully tuning the annealing temperature of as-grown NSC PLCCO, we can control the strength of the AF order and superconductivity without additional complications such as disorder associated with conventional chemical substitution. The temperature dependence of $(0.5,1.5,0)$ shows that the Néel temperature $T_{N}$ decreases linearly with increasing $T_{c}$, and becomes zero at the maximum $T_{c}$ of $24 \mathrm{~K}$. Although the commensurate $\mathrm{AF}$ position $(0.5,0.5,0)$ is forbidden in the noncollinear spin structure of PLCCO (Fig. $2 \mathrm{a})$, clear magnetic scattering is observed at $(0.5,0.5,0)$ 
in underdoped SC PLCCO $\left(T_{c}=21 \mathrm{~K}\right.$ and $\left.16 \mathrm{~K}\right)$ in the $[H, K, 0]$ scattering geometry (Fig. 5). This magnetic component is on top of the structural superlattice peak resulting from the annealing process [33]. Solid lines in Fig. 5 are Gaussian fits to the peaks on sloping backgrounds, where $I=b k g d+I_{0} \exp \left[-\left(H-H_{0}\right)^{2} /\left(2 \sigma^{2}\right)\right]$. Fourier transform of the Gaussian peaks give minimum a in-plane coherence lengths $(C L)$ of $\sim 200 \AA$ for all four samples using $C L=[\sqrt{\ln (2)} / \pi](a / \sigma)$ for $[H, H, 0]$ scans and $C L=[\sqrt{\ln (2) / 5} / \pi](a / \sigma)$ for $[H, 3 H, 0]$ scans. To determine the $c$-axis correlations of the magnetic scattering at $(0.5,0.5,0)$, we aligned the crystal in the $[H, H, L]$ scattering plane but found no large intensity gain centered at $L=0$ in $[0.5,0.5, L]$ scans [36].

If underdoped PLCCO has a quasi-2D SDW with strong correlation in the $\mathrm{CuO}_{2}$ plane but weak coupling along the $c$-axis, the coarse vertical resolution of the triple-axis spectrometer will integrate a much larger region of the $c$-axis magnetic rod in the $[H, K, 0]$ plane than that in the $[H, H, L]$ zone. As a consequence, the quasi$2 \mathrm{D}$ scattering should be more easily observable in the $[H, K, 0]$ zone. The presence of a quasi-2D SDW can be tested using the 2-axis energy integrated mode by aligning the outgoing wave vector $k_{f}$ parallel to the $2 \mathrm{D}$ rod direction (c-axis) (Fig. 4d in Ref. [36]). The temperature dependent scattering around $(0.5,0.5, L)$ in underdoped PLCCO $\left(T_{c}=21\right.$ and $\left.16 \mathrm{~K}\right)$ suggests the presence of quasi-2D SDW modulation (Figs. 6c and 6d) 36]. Because these data were collected using the 2-axis mode, which integrates elastic, inelastic magnetic scattering as well as phonons, its temperature dependence is not a reliable measure of the quasi-elastic diffusive magnetic scattering in Fig. 3 of Ref. [36]. For optimally doped PLCCO $\left(T_{c}=24 \mathrm{~K}\right)$, there is no evidence for the SDW or $3 \mathrm{D} \mathrm{AF}$ order as shown by the temperature independent scattering at $(0.5,0.5,0)$ and $(0.5,1.5,0)$ (Figs. 5a, $5 \mathrm{e}, 6 \mathrm{a}$, and $6 \mathrm{~b})$. For NSC PLCCO, the scattering only shows the static 3D AF order with a small Pr-induced moment at low temperatures [36].

\section{B. Spin-flop transition on superconducting (SC) and nonsuperconducting (NSC) $\operatorname{Pr}_{0.88} \mathrm{LaCe}_{0.12} \mathrm{CuO}_{4 \pm \delta}$}

A magnetic field applied in the $[\overline{1}, 1,0]$ direction of the $\mathrm{CuO}_{2}$ planes induces a spin-flop transition from noncollinear to collinear spin structure in electron-doped parent compounds $\mathrm{Nd}_{2} \mathrm{CuO}_{4}$ and $\mathrm{Pr}_{2} \mathrm{CuO}_{4}$ (Fig. 7) 58 , 59]. For lightly electron-doped $\mathrm{Pr}_{1.29} \mathrm{La}_{0.7} \mathrm{Ce}_{0.01} \mathrm{CuO}_{4 \pm \delta}$, Lavrov et al. discovered that the spin-flop transition is intimately related to the magnetoresistance effect [49]. Similar spin-charge coupling was also found in a separate study on lightly-doped $\mathrm{Nd}_{1.975} \mathrm{Ce}_{0.025} \mathrm{CuO}_{4}$ 60]. The critical field for the transition from a noncollinear spin structure to a collinear one increases with decreasing temperature. For lightly electron-doped $\operatorname{Pr}_{1.29} \mathrm{La}_{0.7} \mathrm{Ce}_{0.01} \mathrm{CuO}_{4 \pm \delta}$, the critical field increases from
TABLE I: Magnetic structure factor calculations for noncollinear $\left(F_{n c}\right)^{2}$ and collinear $\left(F_{c}\right)^{2}$ spin structures as seen in Figures $7 \mathrm{a}$ and $7 \mathrm{~b}$. Here $\gamma e^{2} /\left(2 m c^{2}\right)=0.2695 \times 10^{-12} \mathrm{~cm}$, $f_{c u, p r}, M_{c u, p r}$ are magnetic form factors and ordered magnetic moments for $\mathrm{Cu}^{2+}$ and $\mathrm{Pr}^{3+}$ ions, respectively.

\begin{tabular}{cc}
\hline \hline$(H, K, L)$ & $\left(F_{n c}\right)^{2}$ \\
\hline$(0.5,0.5,0)$ & 0 \\
$(0.5,0.5,1)$ & $32.0\left(\gamma \mathrm{e}^{2} / 2 m \mathrm{c}^{2}\right)^{2}\left(f_{c u} M_{c u}+0.5310 f_{p r} M_{p r}\right)^{2}$ \\
$(0.5,0.5,2)$ & $14.6\left(\gamma \mathrm{e}^{2} / 2 m \mathrm{c}^{2}\right)^{2}\left(f_{c u} M_{c u}+0.2391 f_{p r} M_{p r}\right)^{2}$ \\
$(0.5,0.5,3)$ & $32.0\left(\gamma \mathrm{e}^{2} / 2 m \mathrm{c}^{2}\right)^{2}\left(f_{c u} M_{c u}-0.8196 f_{p r} M_{p r}\right)^{2}$ \\
$(0.5,0.5,4)$ & $24.6\left(\gamma \mathrm{e}^{2} / 2 m \mathrm{c}^{2}\right)^{2}\left(f_{c u} M_{c u}+0.7500 f_{p r} M_{p r}\right)^{2}$ \\
$(0.5,0.5,5)$ & $32.0\left(\gamma \mathrm{e}^{2} / 2 m \mathrm{c}^{2}\right)^{2}\left(f_{c u} M_{c u}-0.0856 f_{p r} M_{p r}\right)^{2}$ \\
\hline \hline & $\left(F_{c}\right)^{2}$ \\
\hline \hline$(H, K, L)$ & 0 \\
$(0.5,0.5,0)$ & 0 \\
$(0.5,0.5,1)$ & 0 \\
$(0.5,0.5,2)$ & $29.2\left(\gamma \mathrm{e}^{2} / 2 m \mathrm{c}^{2}\right)^{2}\left(f_{c u} M_{c u}+0.2391 f_{p r} M_{p r}\right)^{2}$ \\
$(0.5,0.5,3)$ & 0 \\
$(0.5,0.5,4)$ & $49.2\left(\gamma \mathrm{e}^{2} / 2 m \mathrm{c}^{2}\right)^{2}\left(f_{c u} M_{c u}+0.7500 f_{p r} M_{p r}\right)^{2}$ \\
$(0.5,0.5,5)$ & 0 \\
\hline \hline
\end{tabular}

$0.5-\mathrm{T}$ at $150 \mathrm{~K}$ to $2-\mathrm{T}$ at $5 \mathrm{~K}[49]$.

We systematically investigated the effect of an in-plane magnetic field on the spin structure of PLCCO because superconductivity is not much affected in this geometry. The magnetic structure factors at $(0.5,0.5, L)$ for the noncollinear and collinear phases of PLCCO are summarized in Table I. In the collinear spin structure, the magnetic peaks are disallowed at $(0.5,0.5, L)$ with $L=$ odd integer. We probed the $(0.5,0.5,1)$ and $(0.5,0.5,2)$ magnetic peaks as a function of the field at low temperature. Figure 8 summarizes the effect of a $\mathbf{B} \|[\overline{1}, 1,0]$ field on the $(0.5,0.5,1)$ and $(0.5,0.5,2)$ magnetic peaks in different samples of PLCCO at $5 \mathrm{~K}$. The solid lines are Gaussians fits to the data. The $c$-axis coherence lengths are resolution limited and about $660 \AA$ for all three samples estimated using $C L=[\sqrt{2 \ln (2)} / \pi](c / \sigma)$. Therefore, the residual $\mathrm{AF}$ order at $(0.5,0.5, L)(L=1,2, \cdots)$ in underdoped and NSC PLCCO are 3D and different from the quasi-2D diffusive scattering at $(0.5,0.5,0)$.

For the $T_{c}=21 \mathrm{~K}$ PLCCO, a $1-\mathrm{T}$ applied field diminishes the $(0.5,0.5,1)$ and enhances the $(0.5,0.5,2)$ magnetic reflections, thus indicating that the magnetic structure has been transformed from the noncollinear to collinear structure by the field (Figs. 7a-b, 8a and 8d). This sharp transition in the $T_{c}=21 \mathrm{~K} \mathrm{PLCCO}$ is surprising because spin-flop transitions for $\mathbf{B} \|[\overline{1}, 1,0]$ fields in lightly-doped $\operatorname{Pr}_{1.29} \mathrm{La}_{0.7} \mathrm{Ce}_{0.01} \mathrm{CuO}_{4 \pm \delta}$ [49], $\mathrm{Nd}_{1.975} \mathrm{Ce}_{0.025} \mathrm{CuO}_{4}$ [60], $\mathrm{Nd}_{2} \mathrm{CuO}_{4}$ [58], and $\mathrm{Pr}_{2} \mathrm{CuO}_{4}$ 56] are more gradual and continuous.

To understand the evolution of such behavior as PLCCO is transformed from a superconductor to an antiferromagnet, we performed similar measurements on the $T_{c}=16 \mathrm{~K}$ and the NSC PLCCO. The outcome (Figs. 


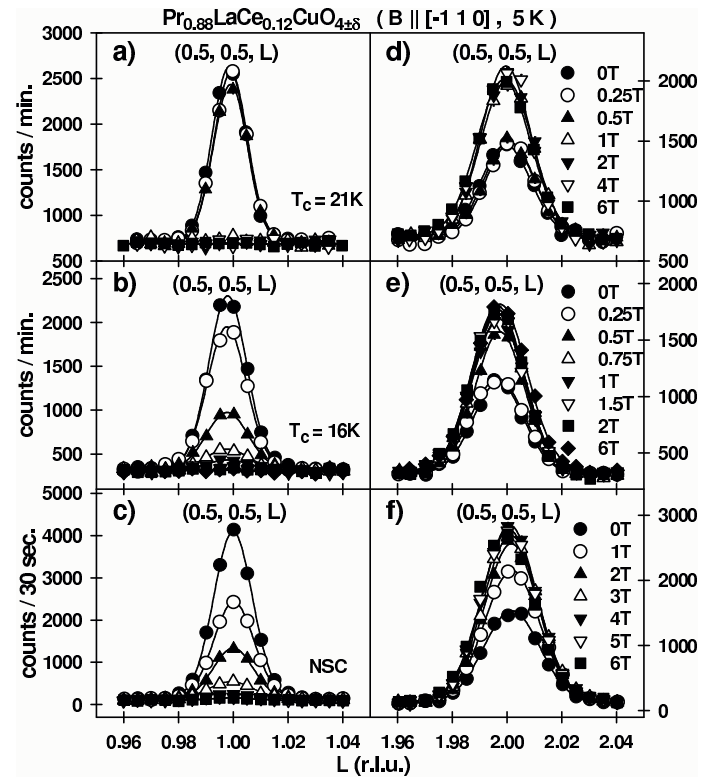

FIG. 8: Magnetic field dependence of the scattering around $(0.5,0.5, L)$ at $5 \mathrm{~K}$, a temperature well below the perspective $T_{N}$ 's for various SC and NSC PLCCO samples. a-c) $L$-scans around $(0.5,0.5,1) \mathrm{d}$-f) $L$-scans around $(0.5,0.5,2)$ for $T_{c}=$ $21 \mathrm{~K}, 16 \mathrm{~K}$ and NSC PLCCO samples. The $T_{c}=21 \mathrm{~K}$ sample shows an abrupt spin-flop transition and the $T_{c}=16 \mathrm{~K}$ and the NSC samples display a more gradual spin-flop transition. The solid lines are Gaussian fits to the data.

$8 \mathrm{~b}$, e, c, and f) suggests that spin-flop transitions become sharper as superconductivity is developed with increasing $T_{c}$ and decreasing $T_{N}$. Figure 9 summarizes the integrated intensities of $(0.5,0.5,1)$ and $(0.5,0.5,2)$ as a function of increasing field for the three PLCCO samples at $5 \mathrm{~K}$. The inset in Fig. 9b shows that a higher Néel temperature $T_{N}$ requires a larger critical field $\mathbf{B}_{s p}$ for the spin-flop transition. $\mathbf{B}_{s p}$ therefore decreases with decreasing $T_{N}$ and reducing $\mathrm{Cu}$ moment.

From the magnetic structure factor calculations in Table I, we find that the ratio of integrated intensities of $(0.5,0.5, L=$ even $)$ at collinear and noncollinear states is $\left[F_{c}(0.5,0.5, L=\text { even }) / F_{n c}(0.5,0.5, L=\text { even })\right]^{2}=2$ if the $\mathrm{Pr}$ and $\mathrm{Cu}$ moments under field are the same as that at zero-field. Inspection of Figs. 8 and 9 for $(0.5,0.5,2)$ shows that this is indeed the case. Therefore, a moderate magnetic field $(\leq 6-\mathrm{T})$ that causes spin-flop transition does not induce additional moments on $\mathrm{Cu}$ or Pr sites in PLCCO.

To further determine the effect of a magnetic field on $\mathrm{Pr} / \mathrm{Cu}$ moments after the spin-flop transition, we measure the temperature dependence of the scattering at $(0.5,0.5, L)(L=2,4,6)$ for fields just above $\mathbf{B}_{s p}$ and at 6 -T. This comparison allows us to determine whether application of additional field induces $\mathrm{Cu} / \mathrm{Pr}$ moments in the collinear state of PLCCO after the spin-flop transition. Inspection of the data in Fig. 10 reveals no appreciable difference between $1.5-\mathrm{T}$ and $6-\mathrm{T}$ in the temperature range probed, consistent with the notion that a 6 - $\mathrm{T}$

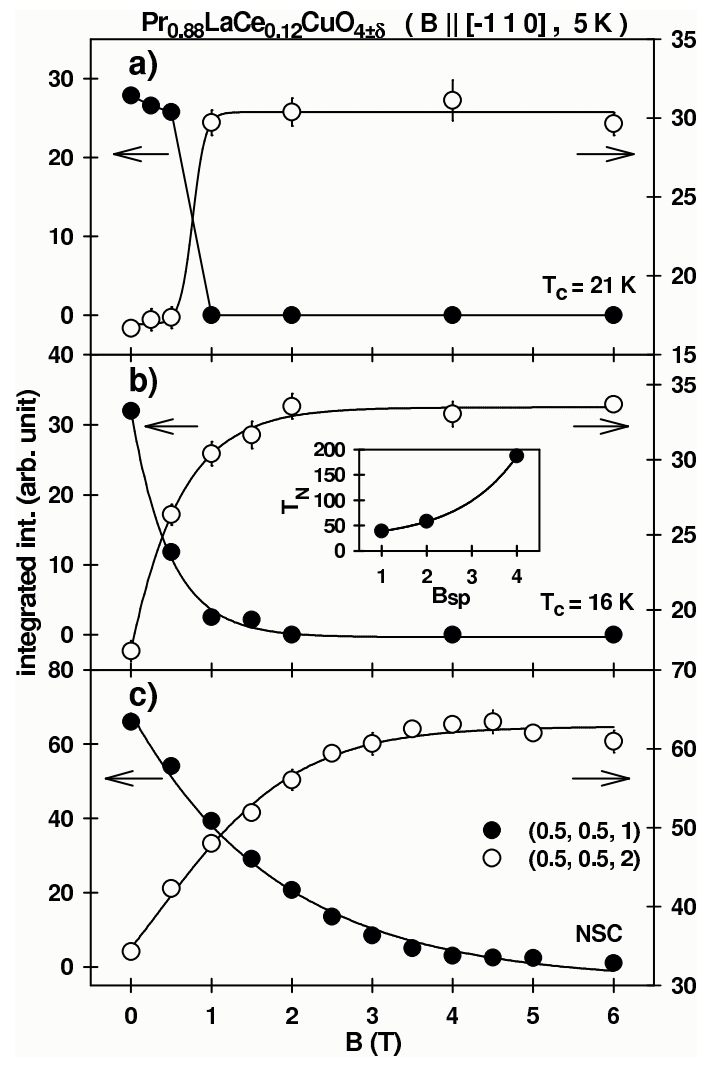

FIG. 9: Integrated intensities of $(0.5,0.5, L)$ as a function of a magnetic field for a) $\left.T_{c}=21 \mathrm{~K}, \mathrm{~b}\right) T_{c}=16 \mathrm{~K}, \mathrm{c}$ ) NSC samples at $5 \mathrm{~K}$. The inset in b) shows that the sample with higher Néel temperature requires a higher critical field to induce the spin-flop transition.

in-plane field does not induce additional $\mathrm{Cu} / \mathrm{Pr}$ moments for the $T_{c}=16 \mathrm{~K}$ PLCCO.

To see if a larger in-plane field will affect the $\mathrm{Cu} / \mathrm{Pr}$ moment, we measured the field-dependence of the $(0.5,0.5,2)$ and $(0.5,0.5,4)$ reflections up to 14 - $\mathrm{T}$ for the $T_{c}=21 \mathrm{~K} \mathrm{PLCCO}$ at HMI. The outcome, shown in Figs. 11c-e, clearly indicates that while a 1-T in-plane field enhances the intensity of the $(0.5,0.5,2)$ peak from that at zero-field as expected from the spin-flop transition, further increasing the applied field to 14 -T does not induce additional changes in the integrated intensity of the $(0.5,0.5,2)$ peak (Fig. 11c). Therefore, a 14-T field does not alter the $\mathrm{Cu} / \mathrm{Pr}$ moments within the uncertainty of our measurements. This is quite different from the effect of an in-plane field on $\mathrm{Nd}_{1.85} \mathrm{Ce}_{0.15} \mathrm{CuO}_{4}$, where the $(0.5,0.5,2)$ reflection shows no intensity differences and the $(0.5,0.5,4)$ intensity decreases under a 7-T field (Figs. 11a-b) [40]. For $\mathrm{Nd}_{1.85} \mathrm{Ce}_{0.15} \mathrm{CuO}_{4}$, a 7-T B $\|[\overline{1}, 1,0]$ magnetic field not only causes a spin-flop transition but also induces magnetic moments on the $\mathrm{Nd}$ sites. The significant intensity drop at $(0.5,0.5,4)$ under 7- $\mathrm{T}$ arises because of the larger $\mathrm{Nd}$ moment contribution to the magnetic structure factor (Table I, for NCCO the Nd moment has opposite direction to Pr moment). 


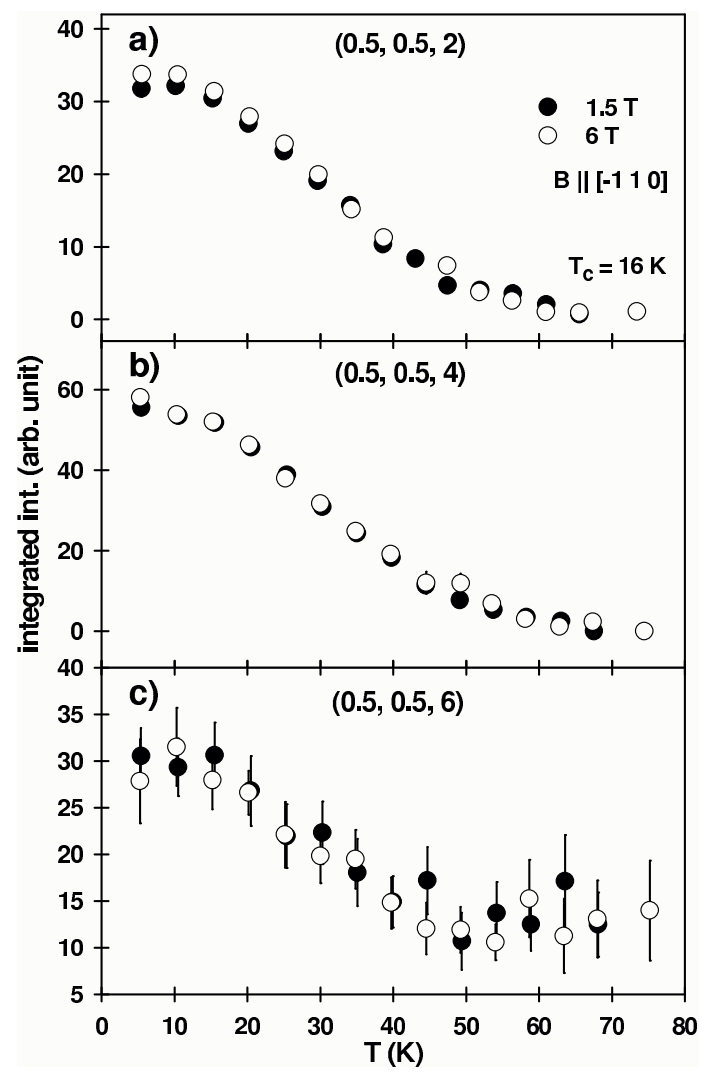

FIG. 10: The temperature dependence of the integrated magnetic Bragg intensities for $(0.5,0.5, L)$ with $L=2,4,6$ at 1.5-T and 6 - $T$ for the $T_{c}=16 \mathrm{~K}$ PLCCO. The spin flop transition should have occurred above 1.5-T (see Fig. 9b). The negligible changes of the integrated intensities indicate that an in-plane magnetic field of 6-T does not affect the $\mathrm{Cu}$ and $\mathrm{Pr}$ moment. It only transforms the magnetic structure from a noncollinear to a collinear one (Fig. 7).

Since PLCCO has negligible field-induced moment contribution from Pr, field-induced effects here should reveal the inherent $\mathrm{Cu}^{2+}$ spin correlations.

\section{Magnetic field effect on the nonsuperconducting (NSC) $\mathbf{P r}_{0.88} \mathrm{LaCe}_{0.12} \mathrm{CuO}_{4 \pm \delta}$ and the cubic $(\mathrm{Pr}, \mathrm{La}, \mathrm{Ce})_{2} \mathrm{O}_{3}$ impurity phase}

Since the discovery of a $c$-axis magnetic field-induced effect in $\mathrm{Nd}_{1.85} \mathrm{Ce}_{0.15} \mathrm{CuO}_{4}$ [4], there has been much debate concerning the origin of the field-induced effect. While we argue that the observed effects are partially intrinsic to $\mathrm{Nd}_{1.85} \mathrm{Ce}_{0.15} \mathrm{CuO}_{4}$ 41, 44, 45, Mang et al. [43, 46] suggest that all the observed field-induced effect in Ref. 40] can be explained by paramagnetic scattering from the impurity $(\mathrm{Nd}, \mathrm{Ce})_{2} \mathrm{O}_{3}$ phase. Since there will always be paramagnetic scattering from $(\mathrm{Nd}, \mathrm{Ce})_{2} \mathrm{O}_{3}$ under the influence of a magnetic field in SC NCCO, it is more productive to study $n$-type superconductors where the impurity phase has no field-induced effect. SC

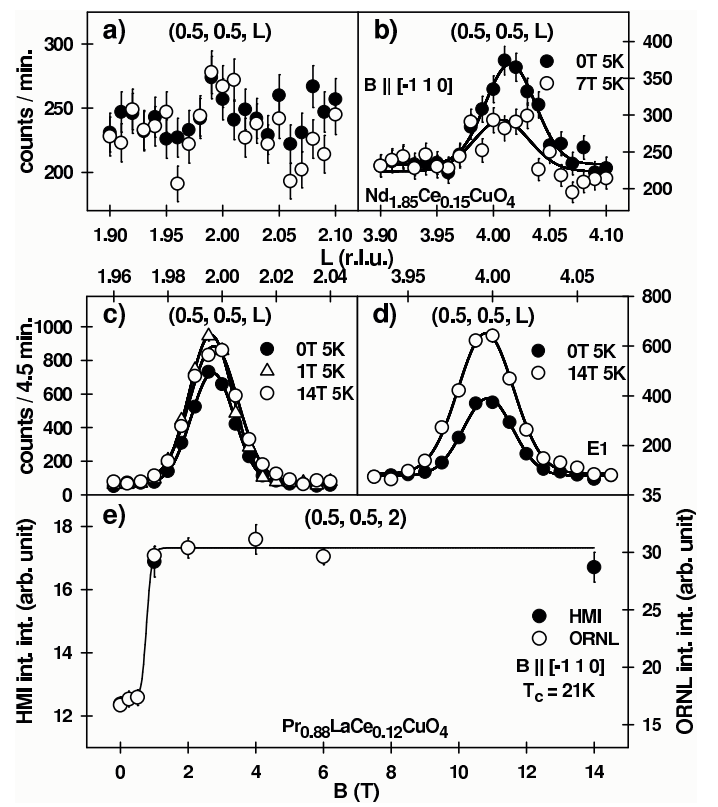

FIG. 11: The comparison of the influence of an in-plane field on the magnetic Bragg peaks of PLCCO and NCCO. a-b) lowtemperature $L$ scans across the magnetic peaks at $(0.5,0.5,2)$ and $(0.5,0.5,4)$ in NCCO (from Ref. [40]). c-d) Similar $L$ scans across $(0.5,0.5,2)$ and $(0.5,0.5,4)$ in PLCCO. The intensity differences between NCCO and PLCCO is due the induced moment on $\mathrm{Nd}^{3+}$ in NCCO. e) integrated intensity of $(0.5,0.5,2)$ as a function of a field. Right after spin-flop transition, the integrated intensity shows no field dependence up to 14-T. This confirms that Pr moment is not induced by a field up to $14-\mathrm{T}$.

PLCCO is a good candidate because $\mathrm{Pr}^{3+}$ in the impurity phase $(\mathrm{Pr}, \mathrm{La}, \mathrm{Ce})_{2} \mathrm{O}_{3}$ has a nonmagnetic singlet ground state. To reveal the intrinsic magnetic field effect on $\mathrm{Cu}^{2+}$ magnetism in SC PLCCO, one must understand the field-induced effect on AF order in NSC PLCCO and impurity $(\mathrm{Pr}, \mathrm{La}, \mathrm{Ce})_{2} \mathrm{O}_{3}$.

To determine the effect of a $c$-axis aligned magnetic field on the AF order of NSC PLCCO, we use the reoxygenated sample $\left(T_{N}=186 \mathrm{~K}\right)$ aligned in the $[H, K, 0]$ scattering plane. Figures $12 \mathrm{a}$ and $\mathrm{b}$ show radial scans across $(0.5,-0.5,0)$ and $(0.5,-1.5,0)$ at zero and 6 - $\mathrm{T} c$ axis aligned fields. To within the error of the measurements, a magnetic field of 6 - $T$ has no effect on either peak at $5 \mathrm{~K}$ although there are small differences in the background scattering of Fig. 12a under field with unknown origin. Since a 6 -T field along the $c$-axis has no effect on NSC $\mathrm{Nd}_{1.85} \mathrm{Ce}_{0.15} \mathrm{CuO}_{4}$ 41], we conclude that such field does not affect the $\mathrm{AF}$ structure or induce moments in NSC PLCCO or $\mathrm{Nd}_{1.85} \mathrm{Ce}_{0.15} \mathrm{CuO}_{4}$.

Next, we consider the influence of a magnetic field on the impurity $(\mathrm{Pr}, \mathrm{La}, \mathrm{Ce})_{2} \mathrm{O}_{3}$ phase. To separate the impurity phase from PLCCO, we aligned the SC PLCCO single crystals in the $[H, H, L]$ zone and applied a magnetic field along the $[\overline{1}, 1,0]$ direction 41, 46]. Since $(\mathrm{Pr}, \mathrm{La}, \mathrm{Ce})_{2} \mathrm{O}_{3}$ has essentially a cubic crystal structure 

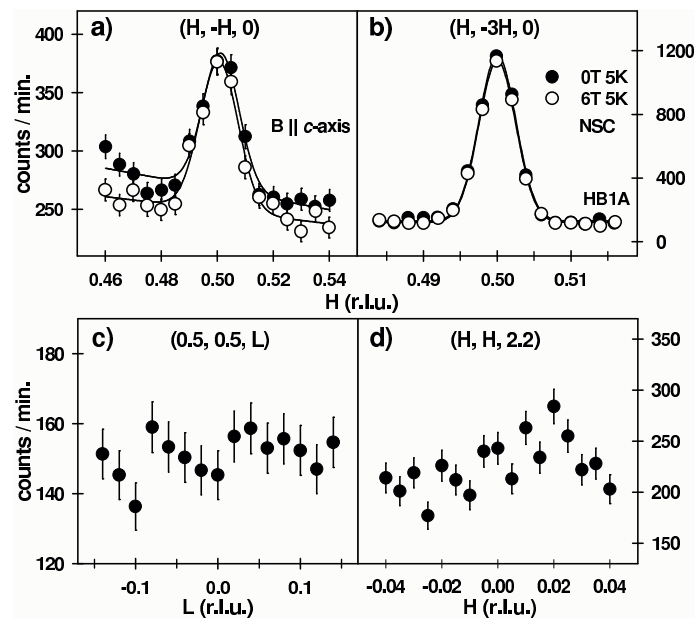

FIG. 12: a-b) Magnetic field dependent scattering around $(0.5,-0.5,0)$ and $(0.5,-1.5,0)$ of NSC PLCCO in the $[H, K, 0]$ scattering plane. Radial scans across a) $(0.5,-0.5,0)$ and $\mathrm{b})(0.5,-1.5,0)$ at $5 \mathrm{~K}$ with zero and 6 - $\mathrm{T}$ fields, respectively. The magnetic field is applied along the $c$-axis. While the scattering at $(0.5,-1.5,0)$ is mostly magnetic from the noncollinear AF order (Fig. 2a), the peak at $(0.5,-0.5,0)$ does not arise from ( $\mathrm{Pr}, \mathrm{La}, \mathrm{Ce})_{2} \mathrm{O}_{3}$ but is diffusive structural scattering of PLCCO similar to those seen in the SC $\mathrm{Nd}_{1.85} \mathrm{Ce}_{0.15} \mathrm{CuO}_{4}$ [41]. This conclusion is confirmed by c) $[0.5,0.5, L]$ and d) $[H, H, 2.2]$ scans across the impurity positions of NSC PLCCO in the $[H, H, L]$ scattering plane.

with lattice parameter $\sim 10 \%$ smaller than the $c$-axis lattice parameter of PLCCO, one can probe the paramagnetic scattering from the impurity phase by simply looking around the $(0,0,2.2)$ position of PLCCO in the $[H, H, L]$ geometry $[41,46]$. Here, cubic reflections $(2,0,0)_{\mathrm{c}}$ and $(0,0,2)_{\mathrm{c}}$ from (Pr,La, Ce $)_{2} \mathrm{O}_{3}$ can be indexed as $(0.5,0.5,0)$ and $(0,0,2.2)$ in PLCCO Miller indices, respectively. A $\mathbf{B} \|[\overline{1}, 1,0]$ field in the $\mathrm{CuO}_{2}$ plane is along the $[1,0,0]$ direction of the cubic $(\mathrm{Pr}, \mathrm{La}, \mathrm{Ce})_{2} \mathrm{O}_{3}$, equivalent to a $c$-axis aligned field in PLCCO which is along the $[0,0,1]$ direction of $(\mathrm{Pr}, \mathrm{La}, \mathrm{Ce})_{2} \mathrm{O}_{3}$.

The field effect on the $(\mathrm{Pr}, \mathrm{La}, \mathrm{Ce})_{2} \mathrm{O}_{3}$ impurity phase was measured in optimally doped and underdoped $\mathrm{SC}$ PLCCO samples with $T_{c}=24 \mathrm{~K}, 21 \mathrm{~K}$, and $16 \mathrm{~K}$. Following previous work on NCCO [41, 43, 46], we probe the impurity peak positions $(0,0,2)_{\mathrm{c}},(1,1,0)_{\mathrm{c}}$, and $(0,0,4)_{\mathrm{c}}$ which correspond to PLCCO Miller indices $(0,0,2.2) /(0.5,0.5,0),(0.5,0,0)$, and $(0,0,4.4)$, respectively. Figure 13 summarizes the outcome around $(0,0,2.2)$ for the three SC PLCCO samples investigated. The $[H, H, 2.2]$ scans across the impurity position at $(0,0,2.2)$ show no observable field-induced effect on the impurity phase up to 14-T (Figs. 13a-c). Additional measurements of the temperature dependent scattering around $(0,0,4.4)$ (Fig. 14a) and the $c$-axis field-dependent scattering around $(0.5,0,0)$ (Fig. 14b) on the $T_{c}=21 \mathrm{~K}$ sample confirm that $\operatorname{Pr}^{3+}$ ions in $(\mathrm{Pr}, \mathrm{La}, \mathrm{Ce})_{2} \mathrm{O}_{3}$ have a nonmagnetic singlet ground state and cannot be polarized by a 14.5 -T field. In contrast,

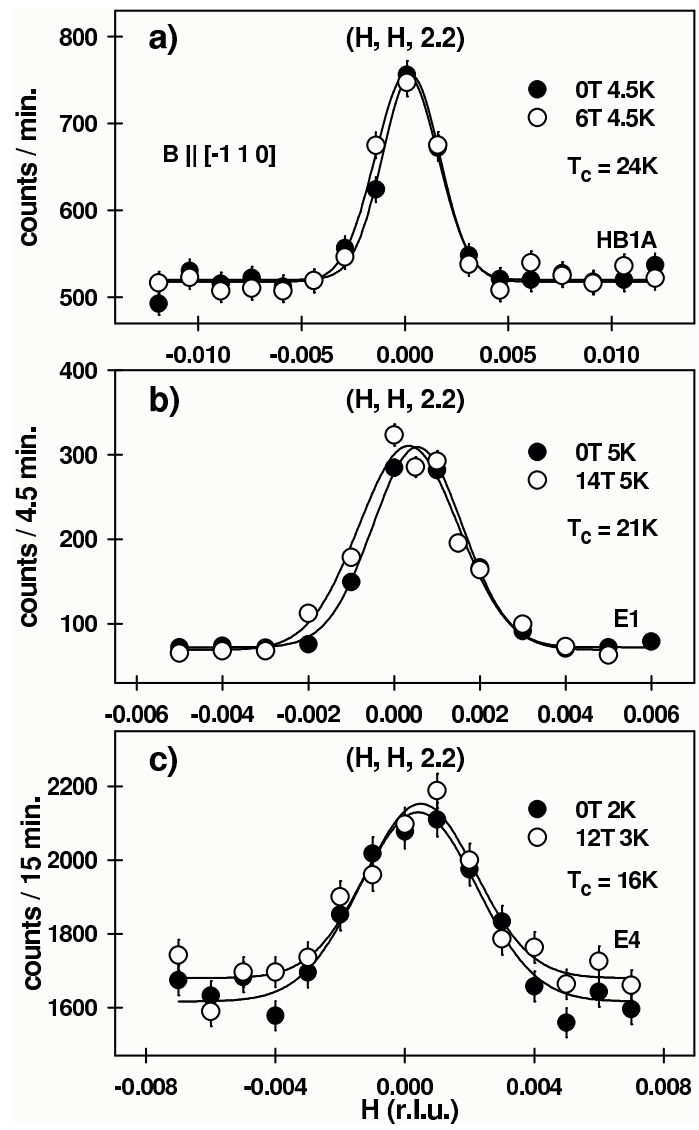

FIG. 13: Magnetic field effect on the impurity peak position $(0,0,2.2)$ at low temperatures. a-c) scans along the $[H, H, 2.2]$ direction for the $T_{c}=24 \mathrm{~K}, 21 \mathrm{~K}$, and $16 \mathrm{~K}$ PLCCO, respectively. The magnetic fields are applied along the $[\overline{1}, 1,0]$ direction, similar to previous work on SC NCCO [41, 43].

$\mathrm{Nd}^{3+}$ ions in the $(\mathrm{Nd}, \mathrm{Ce})_{2} \mathrm{O}_{3}$ impurity phase can be easily polarized by an applied field. Therefore, we can avoid the complication of field-induced paramagnetism from the impurity phase by studying PLCCO and the outcome should unambiguously reveal the intrinsic properties of $\mathrm{Cu}^{2+}$ magnetism in SC electron-doped materials.

\section{Effect of a $c$-axis aligned magnetic field on SDW and AF orders in superconducting (SC) $\operatorname{Pr}_{0.88} \mathrm{LaCe}_{0.12} \mathrm{CuO}_{4 \pm \delta}$}

In this section, we describe the effect of a $c$-axis aligned magnetic field on SC PLCCO samples. Since the previous section confirmed that the $(\mathrm{Pr}, \mathrm{La}, \mathrm{Ce})_{2} \mathrm{O}_{3} \mathrm{impu}-$ rity phase does not respond to an applied magnetic field, any field-induced effect on SC samples must be intrinsic to PLCCO. For the experiment, we aligned crystals in the $[H, K, 0]$ scattering plane and applied magnetic fields along the $c$-axis. Figure 15 shows the radial $[H, H, 0]$ and $[H, 3 H, 0]$ scans around $(0.5,0.5,0)$ and $(0.5,1.5,0)$ positions, respectively, for the SC $T_{c}=24 \mathrm{~K}, 21 \mathrm{~K}$, and $16 \mathrm{~K}$ samples. In all cases, we carefully applied the magnetic 

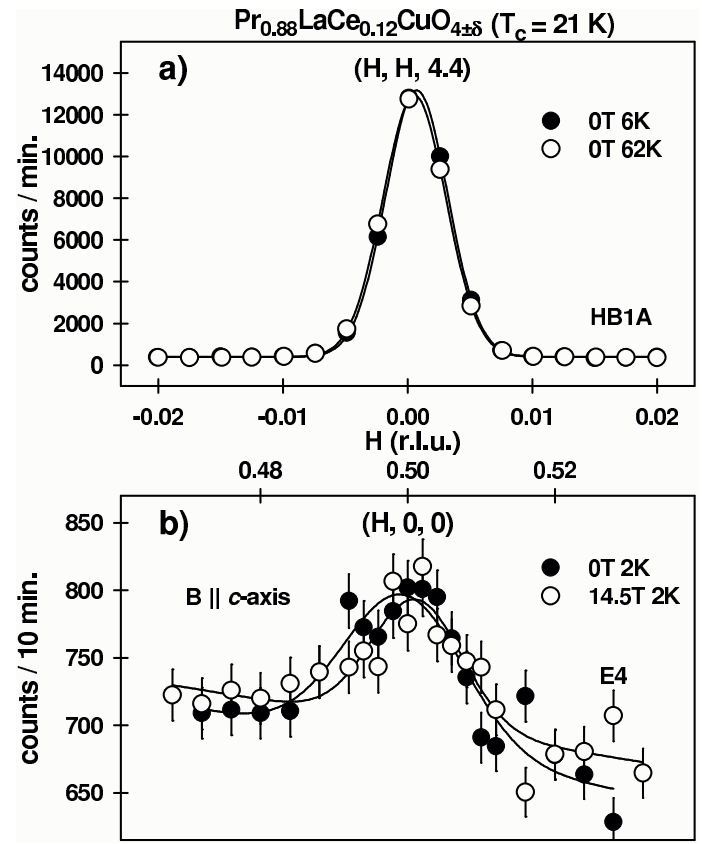

FIG. 14: a) $[H, H, 4.4]$ scan through $(0,0,4.4)$ position for impurity peak $(0,0,4)_{\mathrm{c}}$ at low and high temperatures. The temperature independent scattering indicates that the impurity peak has no magnetic component. b) Radial $[H, 0,0]$ scan across $(0.5,0,0)$ of the impurity peak $(1,1,0)_{\mathrm{c}}$ at zero and 14.5-T $c$-axis aligned fields [45]. It shows no field-induced effect, indicating that the impurity phase cannot be polarized by a $14.5-\mathrm{T}$ field.

field above $T_{c}$ and field-cooled the samples to below $T_{c}$. For optimally doped PLCCO $\left(T_{c}=24 \mathrm{~K}\right)$, a magnetic field of 6.5 - $\mathrm{T}$ at $4.5 \mathrm{~K}$ does not affect the scattering at $(0.5,0.5,0)$ and $(0.5,1.5,0)$ (Fig. 15a and 15e), consistent with an earlier report on an overdoped PLCCO $\left(T_{c}=16\right.$ K) [48]. Note that in this case the peaks at both positions arise mostly from superlattice and $(\mathrm{Pr}, \mathrm{La}, \mathrm{Ce})_{2} \mathrm{O}_{3}$ and there are no static AF orders in the sample (Fig. 6a and $6 \mathrm{~b}$ ) [36].

Underdoped SC samples $\left(T_{c}=21 \mathrm{~K}\right.$ and $\left.16 \mathrm{~K}\right)$, however, show clear low-temperature field-induced intensity gain at the diffusive SDW position $(0.5,0.5,0)$ (Figs. 15bd), but not at the $3 \mathrm{D}$ AF ordered position $(0.5,1.5,0)$ (Figs. 15f-h). In particular, the field-induced intensity appears to increase with increasing magnetic field for the $T_{c}=21 \mathrm{~K} \mathrm{PLCCO}$ (Figs. $15 \mathrm{~b}$ and c). On warming to temperatures above $T_{c}$ but below $T_{N}$, the field-induced effect disappears (Fig. 16). Figure 17 shows the temperature dependence of the scattering at $(0.5,0.5,0)$ and $(0.5,1.5,0)$ for the $T_{c}=21 \mathrm{~K}$ and $16 \mathrm{~K}$ samples at different fields. For the AF 3D ordered Bragg peak $(0.5,1.5,0)$, the intensity simply drops with increasing temperature and shows essentially no difference between field-on and field-off. On the other hand, scattering at $(0.5,0.5,0)$ shows clear low-temperature enhancement under field that vanishes at high temperatures. Although most of the field-induced effects occur at temperatures below the

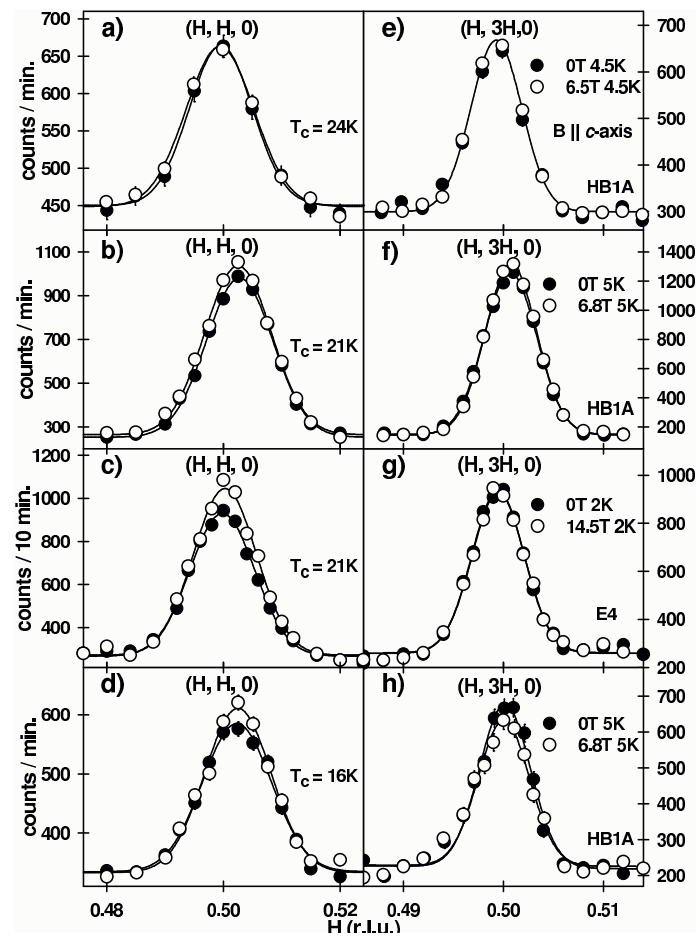

FIG. 15: The effect of a $c$-axis aligned magnetic field on various SC PLCCO samples at low temperatures. The data below 7-T were taken at HB-1A of HFIR while higher field data were collected on E4 of HMI. The $[H, H, 0]$ scans around $(0.5,0.5,0)$ for a) $\left.T_{c}=24 \mathrm{~K}, \mathrm{~b}, \mathrm{c}\right) 21 \mathrm{~K}$, and d) $16 \mathrm{~K}$ PLCCO samples at specified temperatures. e-h) $[H, 3 H, 0]$ scans around $(0.5,1.5,0)$ in the same experimental setup as in a-d).

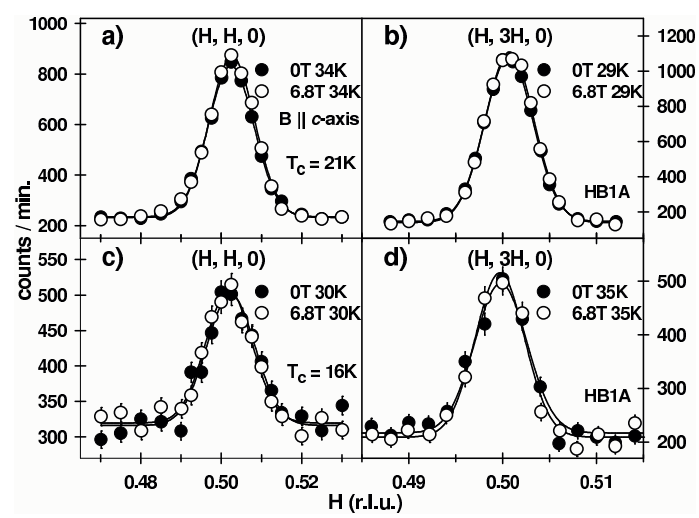

FIG. 16: The field dependence of the scattering across $(0.5,0.5,0)$ and $(0.5,1.5,0)$ at temperatures above $T_{c}$ and below $T_{N}$. a-b) for the $T_{c}=21 \mathrm{~K}$ sample. $\mathrm{c}$-d) for the $T_{c}=16$ $\mathrm{K}$ sample. No field-induced effects are observed.

zero-field $T_{c}$ as marked, we were unable to map out the detailed temperature-field dependence of the effect due to weakness of the signal. This is similar to the fieldinduced effect on incommensurate SDW in LSCO 11] and $\mathrm{La}_{2} \mathrm{CuO}_{4+y}$ [13].

Finally, we carried out a series of $[H, H, 0]$ and $[H, 3 H, 0]$ scans across $(0.5,0.5,0)$ and $(0.5,1.5,0)$ respec- 


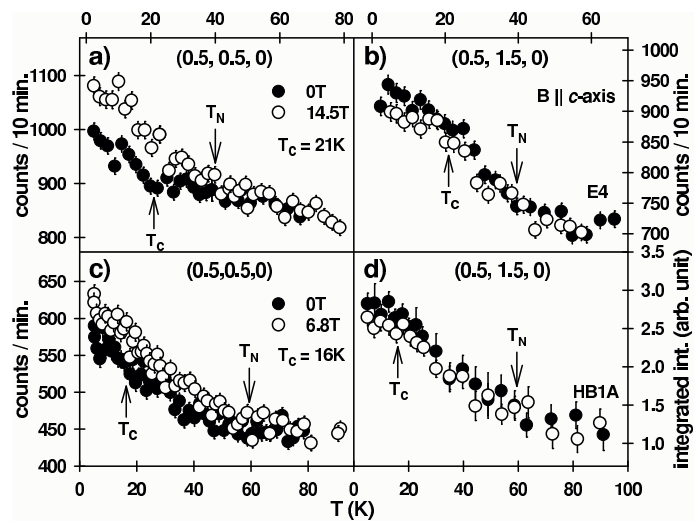

FIG. 17: Temperature dependence of the scattering at the $(0.5,0.5,0)$ and $(0.5,1.5,0)$ positions for $\mathrm{a}-\mathrm{b}) T_{c}=21 \mathrm{~K}$ and c-d) $T_{c}=16 \mathrm{~K}$ samples at zero and finite $c$-axis aligned magnetic fields. Clear low-temperature field-induced effects are seen at $(0.5,0.5,0)$ but not at $(0.5,1.5,0)$ for both the $T_{c}=21$ $\mathrm{K}$ and $16 \mathrm{~K}$ PLCCO samples.

tively for the $T_{c}=21 \mathrm{~K}$ sample to determine the lowtemperature field-dependence of the scattering (Fig. 18). The open circles show the ORNL data taken at $5 \mathrm{~K}$ for fields up to 6.8-T while the filled circles are HMI data at $2 \mathrm{~K}$ for fields up to 14.5-T. The integrated intensity of the SDW at each field is computed by fitting the raw data with a Gaussian on a linear background (See Fig. 15). By combining ORNL and HMI data, we find that the field-induced effect at $(0.5,0.5,0)$ increases linearly with increasing magnetic field up to $13.5 \mathrm{~T}$ and may saturate at higher fields (Fig. 18a). On the other hand, the $3 \mathrm{D} \mathrm{AF}$ residual order $(0.5,1.5,0)$ peak of the $T_{c}=21 \mathrm{PLCCO}$ shows no observable field-induced effect for fields up to 14.5-T (Fig. 18b).

E. Magnetic field-induced anisotropy on the $T_{c}=21$ $\mathrm{K}$ superconducting (SC) $\operatorname{Pr}_{0.88} \mathrm{LaCe}_{0.12} \mathrm{CuO}_{4 \pm \delta}$

The discovery of a $c$-axis field-induced effect unrelated to the (Pr,La,Ce) $)_{2} \mathrm{O}_{3}$ impurity phase in underdoped PLCCO is suggestive but not a proof that such effect is related to the suppression of superconductivity, as one might argue that the AF phase in the SC PLCCO is somehow different from those in NSC samples [46]. One way to check whether the observed effect is indeed related to the suppression of superconductivity is to determine its field directional dependence. Since high- $T_{c}$ superconductors are layered materials, a magnetic field aligned along the $c$-axis suppresses superconductivity much more dramatically than the same field parallel to the $\mathrm{CuO}_{2}$ planes.

To compare the in-plane and $c$-axis magnetic fieldinduced effect, we focus on the SDW at $(0.5,0.5,0)$ for the $T_{c}=21 \mathrm{~K}$ PLCCO. Figure 19 shows scans along the $[H, H, 0]$ and $[0.5,0.5, L]$ directions across $(0.5,0.5,0)$ at zero and $6-\mathbf{T} \mathbf{B} \|[\overline{1}, 1,0]$ in-plane field. There is no

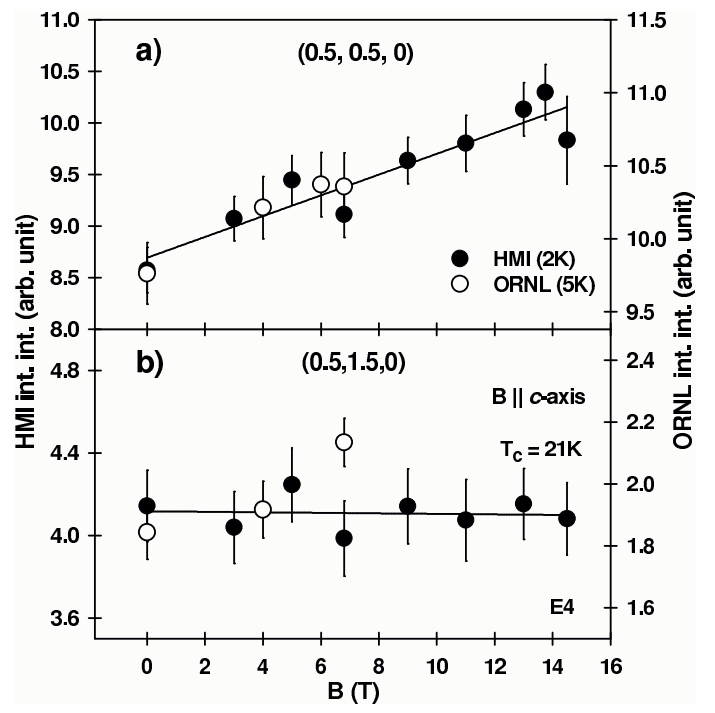

FIG. 18: a) Integrated intensity of $(0.5,0.5,0)$ as a function of increasing field. Open circles are data from ORNL at $5 \mathrm{~K}$ and filled circles are HMI data at $2 \mathrm{~K}$. b) integrated intensity of $(0.5,1.5,0)$ is independent of applied field up to 14.5-T.

observable field-induced effect, in contrast to $c$-axis field data across $(0.5,0.5,0)$ (Fig. 15b-c). On increasing the in-plane applied field to 14-T, we again failed to observe any field-induced effect around $(0.5,0.5,0)$ at $5 \mathrm{~K}$ (Figs. $20 \mathrm{~b}$ and $\mathrm{c})$. For comparison, we replot the data of Fig. $15 \mathrm{c}$ in Fig. 20a. It is clear that a $c$-axis aligned field enhances scattering at $(0.5,0.5,0)$ while the same field parallel to the $\mathrm{CuO}_{2}$ planes does not. Since a 14-T in-plane field only induces the spin-flop transition and does not affect the $\mathrm{Cu} / \mathrm{Pr}$ moments of the residual AF phase, our observation of an anisotropic field effect in the $T_{c}=21 \mathrm{~K}$ PLCCO is the most direct evidence that the enhancement of SDW order is related to the suppression of superconductivity.

The presence of a field-induced effect at the $(0.5,0.5,0)$ SDW position but not at the AF $(0.5,1.5,0)$ Bragg position suggests that $\mathrm{Cu}$ spins contributing to the diffusive SDW cannot arise from the same $\mathrm{Cu}$ spins giving the 3D AF moments. While similar field-induced enhancement was also observed in the case of optimally doped NCCO [40], there are important differences between PLCCO and NCCO. First, optimally doped PLCCO has no residual AF order while $3 \mathrm{D}$ AF order coexists with superconductivity in NCCO even for samples with the highest $T_{c}$ [61]. In addition, there are no detailed studies on how NCCO is transformed from an AF insulator to an optimally doped superconductor. As a consequence, it is unclear how to compare PLCCO directly with NCCO. Second, a $c$-axis aligned field applied on the optimally doped NCCO not only enhances the magnetic signal at $(0.5,0.5,0)$, but also at AF Bragg positions such as $(0.5,1.5,0)$ and $(0.5,0.5,3)$ [41]. For underdoped PLCCO, a 14-T field has no observable effect on $(0.5,1.5,0)$ AF $3 \mathrm{D}$ order. Finally, a $c$-axis aligned field enhances the intensity of the Bragg peak at 

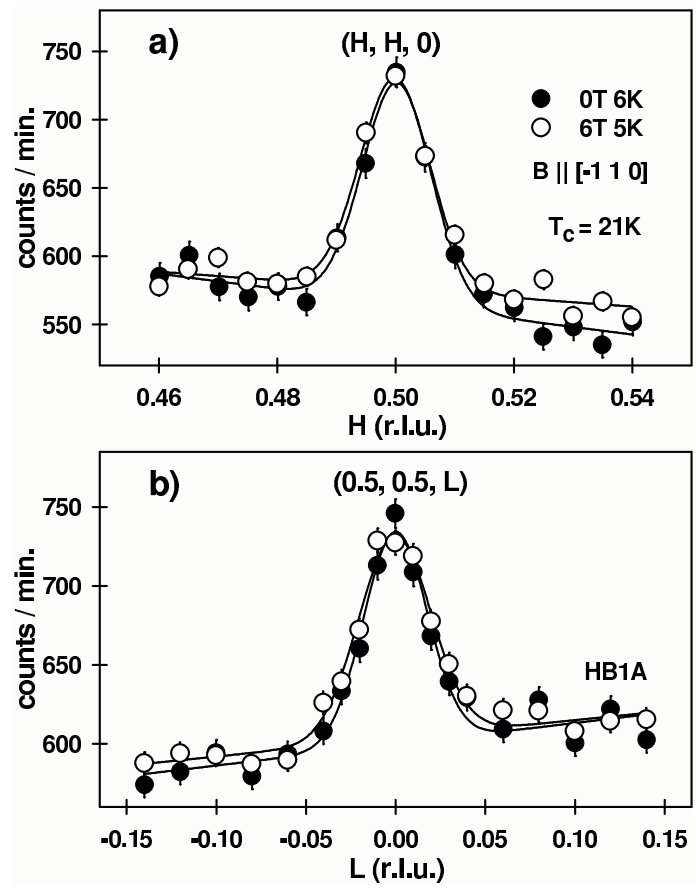

FIG. 19: The influence of an in-plane magnetic field on the SDW order in the $T_{c}=21 \mathrm{~K}$ SC PLCCO. a) $[H, H, 0]$ scan around $(0.5,0.5,0)$ for zero and 6 - $\mathrm{T}$ at $5 \mathrm{~K}$. b) $[0.5,0.5, L]$ scan around $(0.5,0.5,0)$. A 6 - $\mathrm{T}$ in-plane magnetic field has no observable effect on the SDW order at $5 \mathrm{~K}$. The $c$-axis coherence length of $(0.5,0.5,0)$ is about $200 \AA$.

$(1,1,0)$ for both NSC and SC NCCO [41] while a similar field has no observable effect on any nuclear Bragg peaks in NSC and SC PLCCO. This difference must arise from the polarization of the $\mathrm{Nd}^{3+}$ ions in $\mathrm{NCCO}$ and therefore may not be intrinsic to the physics of electron-doped copper oxides.

\section{DISCUSSION}

We use neutron scattering to study the phase transition of PLCCO from a long-range ordered antiferromagnet to a high- $T_{c}$ superconductor without static AF order [36]. In the underdoped regime, we observe the coexistence of a quasi-2D SDW and a 3D AF order in the superconducting state. The $3 \mathrm{D}$ AF order has the same noncollinear magnetic structure as that in undoped $\mathrm{Pr}_{2} \mathrm{CuO}_{4}$ (Fig. 2a). Since the noncollinear spin structure does not allow magnetic Bragg scattering at $(0.5,0.5,0)$, it is interesting to ask whether the $\mathrm{Cu}$ spins giving rise to the $3 \mathrm{D}$ AF order also contribute to the diffusive quasi-2D scattering.

In one picture, the annealing process necessary for producing superconductivity in PLCCO may also induce macroscopic oxygen inhomogeneities, giving rise to mesoscopic separation between the SC and AF NSC phases. In the AF NSC phase, a weak random rotation of the magnetic moment from one plane to another induces a

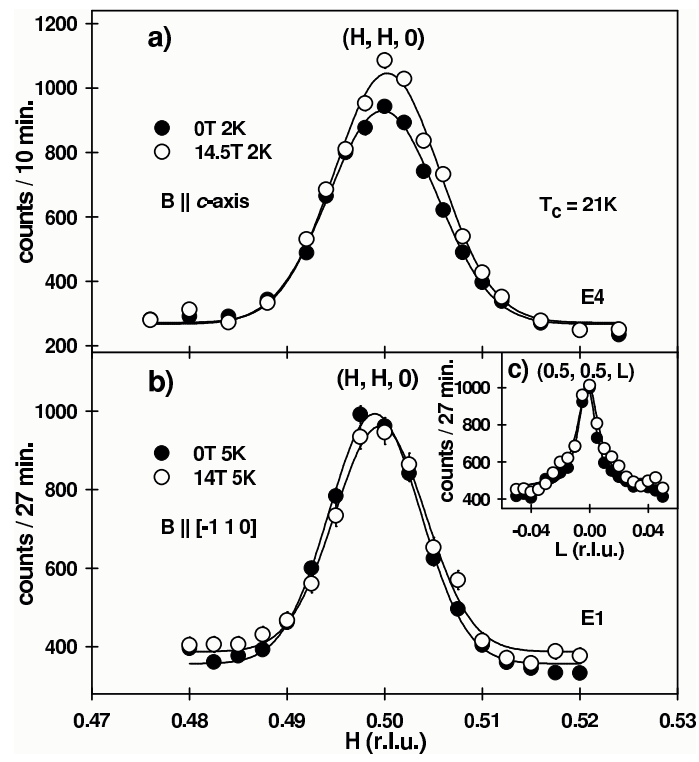

FIG. 20: Anisotropic field-induced effect on the SDW order of the $T_{c}=21 \mathrm{~K}$ PLCCO by a $c$-axis aligned magnetic field and an $a b$-plane magnetic field. a) $[H, H, 0]$ scan across $(0.5,0.5,0)$ with zero and 14.5 - $\mathrm{T}$ field at $2 \mathrm{~K}$ in the $[H, K, 0]$ zone. b) $[H, H, 0]$ scan across $(0.5,0.5,0)$ with a $\mathbf{B} \| a b$-plane magnetic field at zero and $14-\mathrm{T}$. c) $[0.5,0.5, L]$ scan across $(0.5,0.5,0)$ with zero and $14-\mathrm{T}$ at $5 \mathrm{~K}$.

long-range spin order in the plane, but along the $c$-axis one observes simultaneously a Bragg peak and a diffusive scattering. If both the diffusive $(0.5,0.5,0)$ and $3 \mathrm{D} \mathrm{AF}$ $(0.5,1.5,0)$ signals are from the same $\mathrm{Cu}^{2+}$ spins macroscopically phase separated from nonmagnetic SC regions, one would expect that application of a $c$-axis aligned magnetic field will have no (or the same) effect on both the $2 \mathrm{D}$ and $3 \mathrm{D}$ scattering. Instead, we find a clear $c$-axis field-induced effect at $(0.5,0.5,0)$ but not at $(0.5,1.5,0)$ (see section III.D). Therefore, $\mathrm{Cu}^{2+}$ spins contributing to the diffusive scattering cannot arise from the same $\mathrm{Cu}^{2+}$ spins giving $3 \mathrm{D} \mathrm{AF}$ moments.

Alternatively, the annealing process may produce local oxygen distribution fluctuations acting as pinning centers for microscopic electronic phase separation. In this case, the quasi-2D AF scattering may arise from weakly correlated $\mathrm{CuO}_{2}$ layers with a checkerboard $\mathrm{AF}$ order in the matrix of the 3D AF state. The observed diffusive commensurate SDW in PLCCO is then analogous to the incommensurate 2D SDW in hole-doped LSCO and $\mathrm{La}_{2} \mathrm{CuO}_{4+y}$ [4, 5]. When a $c$-axis aligned magnetic field is applied, the residual $3 \mathrm{D} \mathrm{AF}$ order is not disturbed but the diffusive SDW at $(0.5,0.5,0)$ is enhanced at the expense of superconductivity. To estimate an effective moment of the 2D SDW order, we assume two models of spin structures in Fig. 21 that can give magnetic scattering at $(0.5,0.5,0)$. Of course, we don't know the stability of the proposed spin structures in the tetragonal PLCCO unit cell and cannot determine the location of the $\mathrm{Cu}$ spins that contribute the quasi-2D and $3 \mathrm{D}$ AF responses 
TABLE II: Magnetic neutron scattering intensity calculations for proposed spin structures in Fig. 21. The angle $\theta$ is the scattering angle for the Bragg peak. $I_{\text {cal }}(0.5,0.5,0) / I_{\text {cal }}(1,1,0)=I_{o b}(0.5,0.5,0) / I_{o b}(1,1,0)=$ $2.7415 \cdot\left(0.939 M_{c u}\right)^{2} / 5.6531=2.0528 / 4547$ at $0-\mathrm{T}$, and $3.3411 / 4547$ at 14.5 -T using spin structure I.

\begin{tabular}{|c|c|}
\hline$(H, K, L)$ & $I_{\text {model I }}($ domains I \& II $)$ \\
\hline$(0.5,0.5,0)$ & $\frac{1}{2 \sin 2 \theta} 32.0\left(\gamma \mathrm{e}^{2} / 2 m \mathrm{c}^{2}\right)^{2}\left(f_{c u} M_{c u}-0.88 f_{p r} M_{p r}\right)^{2}$ \\
\hline$(0.5,1.5,0)$ & $\frac{1}{2 \sin 2 \theta} 6.4\left(\gamma \mathrm{e}^{2} / 2 m \mathrm{c}^{2}\right)^{2}\left(f_{c u} M_{c u}-0.88 f_{p r} M_{p r}\right)^{2}$ \\
\hline$(H, K, L)$ & $I_{\text {model II }}($ domains I \& II $)$ \\
\hline$(0.5,0.5,0)$ & $\frac{1}{2 \sin 2 \theta} 64.0\left(\gamma \mathrm{e}^{2} / 2 m \mathrm{c}^{2}\right)^{2}\left(f_{c u} M_{c u}-0.88 f_{p r} M_{p r}\right)^{2}$ \\
\hline$(0.5,1.5,0)$ & $\frac{1}{2 \sin 2 \theta} 12.8\left(\gamma \mathrm{e}^{2} / 2 m \mathrm{c}^{2}\right)^{2}\left(f_{c u} M_{c u}-0.88 f_{p r} M_{p r}\right)^{2}$ \\
\hline$(H, K, L)$ & ural in magnetic unit cell \\
\hline$(1,1,0)$ & $\frac{1}{\sin 2 \theta} 64.0\left(0.88 b_{P r}+b_{L a}+0.12 b_{C e}+b_{C u}-4 b_{O}\right)^{2}$ \\
\hline
\end{tabular}

because neutrons are a bulk probe. Our purpose of introducing these models is to estimate an average $\mathrm{Cu}$ moment by normalizing the magnetic intensity at $(0.5,0.5,0)$ to the weak nuclear $(1,1,0)$ reflection. To estimate the effective $\mathrm{Cu}^{2+}$ moment at zero and 14.5-T, we first determine the magnitude of magnetic scattering at zero field by subtacting the integrated intensity of $(0.5,0.5,0)$ at high temperatures from that at $2 \mathrm{~K}$. The total magnetic scattering at $14.5-\mathrm{T}$ is also determined by subtracting the high temperature nonmagnetic structural peak from that at $2 \mathrm{~K}$. For $T_{c}=21 \mathrm{~K} \mathrm{PLCCO}$ at $2 \mathrm{~K}$, the integrated magnetic intensities at zero and $14.5-\mathrm{T}$ are 2.0528 and 3.3411 , respectively. This means that a 14.5 - $\mathrm{T}$ field enhances the magnetic scattering at $(0.5,0.5,0)$ by more than $60 \%[I(14.5-\mathrm{T}) / I(0-\mathrm{T})=1.66]$, much larger than the raw data in Figs. 15 and 16 would suggest. On the same incident beam monitor count, the integrated intensity of $(1,1,0)$ nuclear Bragg peak is 4547.

Assuming each collinear model has two equally populated domains as depicted in Fig. 21 and negligible Pr moment contribution, we can calculate the expected magnetic intensities of these two models in Table II. For the collinear model I with domains I/II (Fig. 21a-b), we obtain the $\mathrm{Cu}$ moment $0.032 \mu_{B}$ at $0-\mathrm{T}$ and $0.041 \mu_{B}$ at 14.5-T. The enhancement of spin correlations appears mostly at $(0.5,0.5,0)$ and the intensity at $(0.5,1.5,0)$ is about 16 times smaller $[I(0.5,1.5,0) / I(0.5,0.5,0)=0.06$, here we used $\theta$ calculated for $E_{i}=E_{f}=13.7 \mathrm{meV}$.]. This is consistent with our observable of a weak (or no) field-induced effect at $(0.5,1.5,0)$. The collinear model II with domains I/II are essentially the same as that of the model I, except the spin directions are along the $[1,1,0]$ and $[\overline{1}, 1,0]$ directions (Figs. 21c-d). This structure gives $\mathrm{Cu}$ moments of $0.022 \mu_{B}$ at $0-\mathrm{T}$ and $0.028 \mu_{B}$ at $14.5-$ $\mathrm{T}$. Therefore, the field-induced enhancement of the $\mathrm{Cu}$ moment is smaller $\left(\sim 0.006 \mu_{B}\right)$ than that of hole-doped LSCO and LCO in these two models [1, 12].

When as-grown PLCCO is transformed from a longrange ordered antiferromagnet to a high- $T_{c}$ superconductor by annealing in pure Ar, the 3D AF order is degraded

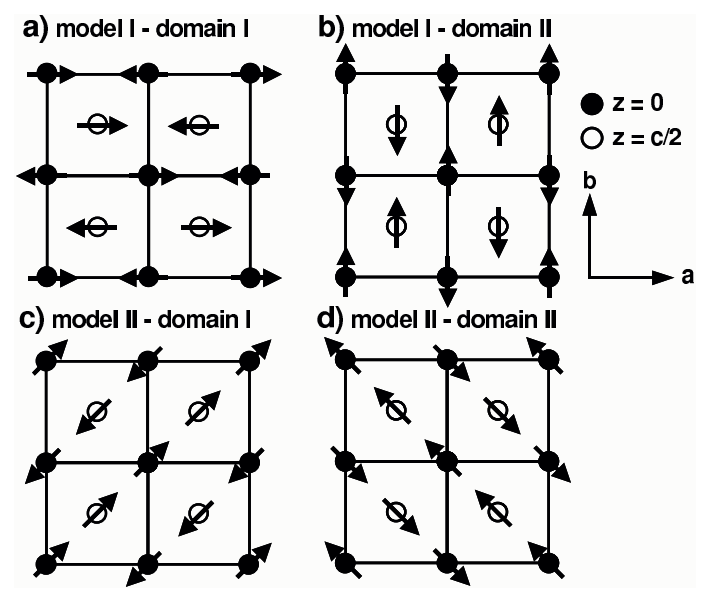

FIG. 21: Possible $\mathrm{Cu}^{2+}$ magnetic structures responsible for the SDW order. a-b) Collinear spin model I, domains I and II. c-d) collinear spin model II, domains I and II. In collinear spin structures, the moments are in the $a b$-plane. The closed and open circles represent the $z=0$ and $z=c / 2 \mathrm{CuO}_{2}$ planes, respectively. The $\mathrm{Pr}^{3+}$ spins are not shown, since the induced moment of $\mathrm{Pr}^{3+}$ is negligible in the SC PLCCO samples. Here we assumed weak (2 or 3 lattices) spin correlations along the $c$-axis. Experimentally, we probably cannot distinguish such scattering from that of random $2 \mathrm{D}$ sheets in the $3 \mathrm{D}$ AF matrix.

and a quasi-2D SDW order develops with the appearance of superconductivity. As optimal superconductivity is reached with $T_{c}=24 \mathrm{~K}$, both static $3 \mathrm{D} A F$ and diffusive SDW orders vanish. We confirm that the annealing process necessary for producing superconductivity also induces the bixbyite ( $\mathrm{Pr}, \mathrm{La}, \mathrm{Ce})_{2} \mathrm{O}_{3}$ as an impurity phase. However, in contrast to $(\mathrm{Nd}, \mathrm{Ce})_{2} \mathrm{O}_{3}$ in $\mathrm{SC} \mathrm{NCCO}$, a 14$\mathrm{T}$ field cannot polarize $\mathrm{Pr}^{3+}$ in $(\mathrm{Pr}, \mathrm{La}, \mathrm{Ce})_{2} \mathrm{O}_{3}$ and this property therefore allows an unambiguous identification of the intrinsic field-induced effect on PLCCO. At optimal doping, a 6.5-T $c$-axis aligned field does not induce static AF order due to the presence of a spin gap [48]. For underdoped materials, a 14-T $c$-axis field enhances the SDW at $(0.5,0.5,0)$ but has no observable effect on $3 \mathrm{D}$ residual $\mathrm{AF}$ order at $(0.5,1.5,0)$. A similar field applied along the $[\overline{1}, 1,0]$ direction in the $\mathrm{CuO}_{2}$ plane only induces a spin-flop transition but has no effect on the SDW order. This is the most direct evidence that the enhancement of SDW is associated with the suppression of superconductivity.

While our results for optimally doped PLCCO are consistent with that for an overdoped PLCCO by Fujita et al. 48], we cannot confirm their results for the $x=0.11$ $\left(T_{c}=25 \mathrm{~K}\right)$ PLCCO. In particular, the 3D AF order has no observable field dependence in our underdoped samples. On the other hand, it would also be interesting to check if their $x=0.11$ PLCCO sample has a field-induced effect at $(0.5,0.5,0)$. At present, it is unclear how to reconcile our results with that of Fujita et al. [48]. Perhaps the differences in Ce concentration and/or post annealing oxygen treatment plays an important role in determin- 
TABLE III: Magnetic structure factor calculation for noncollinear spin structure of NCCO (type I/III) and PLCCO.

\begin{tabular}{cc}
\hline \hline$(H, K, L)$ & $(F)^{2}$ for NCCO type I/III \\
\hline$(0.5,1.5,0)$ & $25.6\left(\gamma \mathrm{e}^{2} / 2 m \mathrm{c}^{2}\right)^{2}\left(f_{c u} M_{c u}+1.85 f_{n d} M_{n d}\right)^{2}$ \\
$(0.5,0.5,3)$ & $32.0\left(\gamma \mathrm{e}^{2} / 2 m \mathrm{c}^{2}\right)^{2}\left(f_{c u} M_{c u}+1.7426 f_{n d} M_{n d}\right)^{2}$ \\
\hline \hline$(H, K, L)$ & $(F)^{2}$ for PLCCO \\
\hline$(0.5,1.5,0)$ & $25.6\left(\gamma \mathrm{e}^{2} / 2 m \mathrm{c}^{2}\right)^{2}\left(f_{c u} M_{c u}-0.88 f_{p r} M_{p r}\right)^{2}$ \\
$(0.5,0.5,3)$ & $32.0\left(\gamma \mathrm{e}^{2} / 2 m \mathrm{c}^{2}\right)^{2}\left(f_{c u} M_{c u}-0.8196 f_{p r} M_{p r}\right)^{2}$ \\
\hline \hline
\end{tabular}

ing the properties of these materials. In any case, what is clear is that underdoped PLCCO has a field-induced effect unrelated to the impurity phase, and such an effect appears to be associated with the suppression of superconductivity [42].

To determine the influence of an applied field on the residual 3D AF order in $\mathrm{SC}$ electron-doped cuprates, one must understand the differences in the field-induced effect between optimally doped NCCO and underdoped PLCCO. The optimally doped NCCO shows clear $c$ axis field-induced effects at the $3 \mathrm{D}$ AF ordering positions such as $(0.5,1.5,0)$ and $(0.5,0.5,3)$. On the other hand, we were unable to find clear field-induced effects at $(0.5,1.5,0)$ for underdoped PLCCO. This difference may arise from the different rare-earth moment contributions to the magnetic scattering. For NCCO, the structure factor calculations in Table III show that the enhanced $\mathrm{Nd}^{3+}$ moment from the exchange coupling to the field-induced $\mathrm{Cu}^{2+}$ moment contributes constructively to the $(0.5,1.5,0)$ and $(0.5,0.5,3)$ reflections. On the other hand, the $\mathrm{Pr}^{3+}$ induced moment contributes destructively to the $\mathrm{Cu}^{2+}$ moment in PLCCO. As a consequence, any field-induced $\mathrm{Pr}^{3+}$ moment should reduce the scattering intensity at $(0.5,1.5,0)$ for PLCCO while a small $\mathrm{Cu}^{2+}$ moment enhancement will induce large intensity enhancement at $(0.5,1.5,0)$ and $(0.5,0.5,3)$ for NCCO. Since SC PLCCO has a negligible field-induced $\mathrm{Pr}^{3+}$ moment, a small field-induced enhancement in the $\mathrm{Cu}^{2+}$ moment may not be easily observable at $(0.5,1.5,0)$.

Our results can be thought of as analogous to that in hole-doped LSCO. When superconductivity first emerges in LSCO with increasing Sr-doping, quasi-2D incommensurate SDW peaks also appear spontaneously to coexist with superconductivity as shown in Fig. 1b [7, 8, 9]. As LSCO becomes an optimally doped superconductor, the static SDW is replaced by a spin gap and incommensurate spin fluctuations at energies above the gap [6]. A $c$-axis aligned magnetic field that strongly suppresses superconductivity also enhances the static SDW order in underdeopd LSCO 10, 11] and induces states within the gap in optimally doped LSCO 14, 15]. Very recently, Khaykovich et al. 62] report the observation of a magnetic-field-induced transition between magnetically disordered and ordered phases in slightly underdoped LSCO with $x=0.144$. Here, static incommensurate SDW order can be induced directly by a field from the sample without zero-field SDW order.

For electron-doped PLCCO, we also find the simultaneous appearance of a quasi-2D SDW order and superconductivity, except in this case the SDW modulations are commensurate with the underlying lattice (Fig. 1a) [36]. Similar to LSCO, optimally doped PLCCO also does not exhibit static SDW order. Work is currently underway to determine the size of the spin gap and spin fluctuations at high energies. When a $c$-axis aligned magnetic field is applied, the static commensurate SDW order is enhanced for underdoped PLCCO but not for optimally doped samples. These results are very similar to hole-doped LSCO, thus suggesting the universality of the magnetic properties in both hole- and electron-doped cuprates. In the coming years, neutron scattering experiments will be carried out to search for the spin excitations in optimally and underdoped PLCCO. Our ultimate goal is to determine whether magnetism plays a fundamental role in controlling the properties of doped cuprates and the mechanism of high- $T_{c}$ superconductivity

\section{SUMMARY AND CONCLUSIONS}

We have systematically investigated the effect of a magnetic field on electron-doped PLCCO materials from an AF insulator to an optimally doped superconductor. By controlling the annealing temperatures, we obtain PLCCO samples with different $T_{c}$ 's and $T_{N}$ 's. When superconductivity first appears in PLCCO, a quasi-2D SDW order is also induced at $(0.5,0.5,0)$, and both coexist with the residual 3D AF state. While the annealing process also induces cubic $(\mathrm{Pr}, \mathrm{La}, \mathrm{Ce})_{2} \mathrm{O}_{3}$ as an impurity phase similar to $(\mathrm{Nd}, \mathrm{Ce})_{2} \mathrm{O}_{3}$ in $\mathrm{SC} \mathrm{NCCO}$, we show that a magnetic field up to 14- $\mathrm{T}$ does not induce magnetic scattering in this impurity phase. To determine whether $\mathrm{AF}$ order is a competing ground state for superconductivity in electron-doped superconductors, we first must understand the influence of a magnetic field on the residual AF order for SC and NSC PLCCO without the complication of superconductivity. We confirm that a $c$-axis aligned magnetic field has no effect on the $\mathrm{AF}$ order in the NSC PLCCO. Because PLCCO is a layered superconductor with highly anisotropic $B_{c 2}$, superconductivity can be dramatically suppressed by a $\mathbf{B} \| c$-axis field but much less affected by the same field in the $\mathrm{CuO}_{2}$ plane. Utilizing this property, we determine the influence of an in-plane magnetic field on the residual AF order in $\mathrm{SC}$ PLCCO. We find that a 14 -T field along the $[\overline{1}, 1,0]$ direction only causes a spin-flop transition but does not induce additional moments on $\mathrm{Cu}^{2+}$ and/or $\mathrm{Pr}^{3+}$ site in the SC PLCCO's. This suggests that the residual AF order in SC PLCCO behaves in a similar way as AF order in NSC materials.

To study the magnetic field effect on SDW modulations in SC PLCCO, we conducted experiments in two ways. First, we aligned the crystal in the $[H, K, 0]$ geometry and applied magnetic field along the $c$-axis. For 
both $T_{c}=21 \mathrm{~K}$ and $16 \mathrm{~K}$ PLCCO samples, the $c$-axis aligned field enhances the SDW order at the $(0.5,0.5,0)$ position but has no observable effect at the $3 \mathrm{D}$ AF order position $(0.5,1.5,0)$. The field-induced enhancement at $(0.5,0.5,0)$ increases with increasing magnetic field up to 13.5 - $\mathrm{T}$ for the $T_{c}=21 \mathrm{~K}$ sample. The $T_{c}=16 \mathrm{~K}$ sample also shows a field-induced effect at $(0.5,0.5,0)$ for measured fields up to $6.8-T$. Second, the crystal was aligned in the $[H, H, L]$ scattering plane and a magnetic field was applied along the $[\overline{1}, 1,0]$ direction. In this scattering geometry, both $[0.5,0.5, L]$ and $[H, H, 0]$ radial scans around the $(0.5,0.5,0)$ position in the $\mathrm{CuO}_{2}$-plane show no observable field-induced effect. This anisotropy of a field effect confirms that the enhancement of SDW in the $c$-axis field direction is related to the suppression of su- perconductivity.

\section{ACKNOWLEDGMENTS}

We are grateful to M. Matsuura, Jerel Zarestky, and K. Prokes for technical assistance with our neutron scattering measurements at ORNL and HMI, respectively. We would also like to acknowledge Branton Campbell, M. Matsuura, Stephan Rosenkranz, J. M. Tranquada, S. C. Zhang, and K. Yamada for helpful discussions. This work is supported by the U. S. NSF DMR-0139882, DOE No. DE-AC05-00OR22725 with UT/Battelle, LLC.
[1] M. A. Kastner, R. J. Birgeneau, G. Shirane, and Y. Endoh, Rev. Mod. Phys. 70, 897 (1998).

[2] S. Sachdev and S.-C. Zhang, Science 295, 452 (2002).

[3] B. G. Levi, Physics Today 57, 24 (2004).

[4] H. Kimura, K. Hirota, H. Matsushita, K.Yamada, Y. Endoh, S-H Lee, C. F. Majkrzak, R. Erwin, M. Greven, Y. S. Lee, M. A. Kastner, and R. J. Birgeneau, Phys. Rev. B 59, 6517 (1999).

[5] Y. S. Lee, R. J. Birgeneau, M. A. Kastner, Y. Endoh, S. Wakimoto, K. Yamada, R. W. Erwin, S.-H. Lee, and G. Shirane, Phy. Rev. B 60, 3643 (1999).

[6] K. Yamada, C. H. Lee, K. Kurahashi, J. Wada, S. Wakimoto, S. Ueki, H. Kimura, Y. Endoh, S. Hosoya, G. Shirane, R. J. Birgeneau, M. Greven, M. A. Kastner, and Y. J. Kim, Phys. Rev. B 57, 6165 (1998).

[7] S. Wakimoto, G. Shirane, Y. Endoh, K. Hirota, S. Ueki, K. Yamada, R. J. Birgeneau, M. A. Kastner, Y. S. Lee, P. M. Gehring, and S. H. Lee, Phys. Rev. B 60, R769 (1999).

[8] M. Fujita, K. Yamada, H. Hiraka, P. M. Gehring, S. H. Lee, S. Wakimoto, and G. Shirane, Phys. Rev. B 65, 064505 (2002).

[9] M. Matsuda, M. Fujita, K. Yamada, R. J. Birgeneau, Y. Endoh, and G. Shirane, Phys. Rev. B 65, 134515 (2002).

[10] S. Katano, M. Sato, K. Yamada, T. Suzuki, and T. Fukase, Phy. Rev. B 62, R14677 (2000).

[11] B. Lake, H. M. Ronnow, N. B. Christensen, G. Aeppli, K. Lefmann, D. F. McMorrow, P. Vorderwisch, P. Smeibidl, N. Mangkorntong, T. Sasagawa, M. Nohara, H. Takagi, and T. E. Mason, Nature 415, 299 (2002)

[12] B. Khaykovich, Y. S. Lee, R. W. Erwin, S.-H. Lee, S. Wakimoto, K. J. Thomas, M. A. Kastner, and R. J. Birgeneau, Phy. Rev. B 66, 014528 (2002).

[13] B. Khaykovich, R. J. Birgeneau, F. C. Chou, R. E. Erwin, M. A. Kastner, S.-H. Lee, Y. S. Lee, P. Smeibidl, P. Vorderwisch, and S. Wakimoto, Phys. Rev. B 67, 054501 (2003).

[14] B. Lake, G. Aeppli, K. N. Clausen, D. F. McMorrow, K. Lefmann, N. E. Hussey, N. Mangkorntong, N. Nahara, H. Takagi, and T. E. Mason, Science 291, 1759 (2001).

[15] J. M. Tranquada, C. H. Lee, K. Yamada, Y. S. Lee, L. P. Regnault, H. M. Ronnow, Phys. Rev. B 69, 174507 (2004).
[16] B. Lake, K. Lefmann, N. B. Christensen, G. Aeppli, D. F. McMorrow, P. Vorderwisch, P. Smeibidl, N. Mangkorntong, T. Sasagawa, M. Nohara, H. Takagi, (unpublished).

[17] Shou-Cheng Zhang, Science 275, 1089 (1997).

[18] Daniel P. Arovas, A. J. Berlinsky, C. Kallin, and ShouCheng Zhang, Phy. Rev. Lett. 79, 2871 (1997).

[19] Y. Zhang, E. Demler, and S. Sachdev, Phys. Rev. B 66, 094501 (2002).

[20] D.-H. Lee, Phys. Rev. Lett. 88, 227003 (2002).

[21] Y. Chen and C. S. Ting, Phys. Rev. B 65, 180513 (2002).

[22] S. A. Kivelson, D.-H. Lee, E. Fradkin, and O. Oganesyan, Phys. Rev. B 66, 144516 (2002).

[23] J. Zhu, I. Martin, and A. Bishop, Phys. Rev. Lett. 89, 067003 (2002).

[24] E. Demler, W. Hanke, and S.-C. Zhang, Rev. Mod. Phys. 76, 909 (2004).

[25] Pengcheng Dai, H. A. Mook, G. Aeppli, S. M. Hayden, and F. Doğan, Nature (London) 406, 965 (2000).

[26] H. A. Mook, Pengcheng Dai, S. M. Hayden, A. Hiess, J. W. Lynn, S.-H. Lee, and F. Doğan, Phys. Rev. B 66, 144513 (2002).

[27] Y. Hidaka and M. Suzuki, Nature (London) 338, 635 (1989).

[28] P. Fournier, P. Mohanty, E. Maiser, S. Darzens, T. Venkatesan, C. J. Lobb, G. Czjzek, R. A. Webb, and R. L. Greene, Phys. Rev. Lett. 81, 4720 (1998).

[29] Y. Wang, S. Ono, Y. Onose, G. Gu, Y. Ando, Y. Tokura, S. Uchida, and N. P. Ong, Science 299, 86 (2003).

[30] Y. Tokura, H. Takagi, and S. Uchida, Nature (London) 337, 345 (1989).

[31] H. Takagi, S. Uchida, and Y. Tokura, Phys. Rev. Lett. 62, 1197 (1989).

[32] J. S. Kim and D. R. Gaskell, Physica C 209, 381 (1993).

[33] K. Kurahashi, H. Matsushita, M. Fujita, and K. Yamada, J. Phys. Soc. Jpn. 71, 910 (2002).

[34] M. Fujita, T. Kubo, S. Kuroshima, T. Uefuji, K. Kawashima, K. Yamada, I. Watanable, and K. Nagamine, Phys. Rev. B 67, 014514 (2003).

[35] Here we call the observed quasi-2D AF scattering spin density wave (SDW) because of its similarity to the quasi2D AF scattering seen in hole-doped materials [4, 5].

[36] Pengcheng Dai, H. J. Kang, H. A. Mook, M. Matsuura, J. W. Lynn, Y. Kurita, S. Komiya, and Y. Ando, 
cond-mat/0501120 (2005).

[37] K. Yamada, K. Kurahashi, Y. Endoh, R. J. Birgeneau, and G. Shirane, J. Phys. and Chem. Solids 60, 1025 (1999).

[38] K. Yamada, K. Kurahashi, T. Uefuji, M. Fujita, S. Park, S.-H. Lee, and Y. Endoh, Phys. Rev. Lett. 90, 137004 (2003).

[39] M. Matsuda, S. Katano, T. Uefuji, M. Fujita, and K. Yamada, Phys. Rev. B 66, 172509 (2002).

[40] H. J. Kang, Pengcheng Dai, J. W. Lynn, M. Matsuura, J. R. Thompson, Shou-Cheng Zhang, D. N. Argyriou, Y. Onose, and Y. Tokura, Nature 423, 522 (2003).

[41] M. Matsuura, Pengcheng Dai, H. J. Kang, J. W. Lynn, D. N. Argyriou, K. Prokes, Y. Onose, and Y. Tokura, Phys. Rev. B 68, 144503 (2003).

[42] Han-Dong Chen, Congjun Wu, and Shou-Cheng Zhang, Phys. Rev. Lett. 92, 107002 (2004).

[43] P. K. Mang, S. Larochelle, and M. Greven, Nature 426, 139 (2003).

[44] H. J. Kang, Pengcheng Dai, J. W. Lynn, M. Matsuura, J. R. Thompson, Shou-Cheng Zhang, D. N. Argyriou, Y. Onose, and Y. Tokura, Nature 426, 140 (2003).

[45] M. Matsuura, Pengcheng Dai, H. J. Kang, J. W. Lynn, D. N. Argyriou, Y. Onose, and Y. Tokura, Phys. Rev. B 69, 104510 (2004).

[46] P. K. Mang, S. Larochelle, A. Mehta, O. P. Vajk, A. S. Erickson, L. Lu, W. J. L. Buyers, A. F. Marshall, K. Prokes, and M. Greven, Phys. Rev. B 70, 094507 (2004).

[47] A. T. Boothroyd, S. M. Doyle, D. Mck. Paul, and R. Osborn, Phys. Rev. B 45, 10075 (1992).

[48] M. Fujita, M. Matsuda, S. Katano, and K. Yamada, Phys. Rev. Lett. 93, 147003(2004).

[49] A. N. Lavrov, H. J. Kang, Y. Kurita, T. Suzuki, Seiki Komiya, J. W. Lynn, S.-H. Lee, Pengcheng Dai, and Yoichi Ando, Phys. Rev. Lett. 92, 227003 (2004).

[50] X. F. Sun, Y. Kurita, T. Suzuki, Seiki Komiya, and Yoichi Ando, Phys. Rev. Lett. 92, 047001 (2004).

[51] Wu Jiang, J. L. Peng, Z. Y. Li, and R. L. Greene, Phys.
Rev. B 47, 8151 (1993).

[52] P. G. Radaelli, J. D. Jorgensen, A. J. Schultz, J. L. Peng, and R. L. Greene, Phys. Rev. B 49, 15322 (1994).

[53] A. Nath, N. S. Kopelev, V. Chechersky, J. L. Peng, R. L. Greene, O. Boem-hoan, M. I. Larkin, and J. T. Markert, Science 265, 73 (1994).

[54] G. Riou, P. Richard, S. Jandl, M. Poirier, P. Fournier, V. Nekvasil, S. N. Barilo, and L. A. Kurnevich, Phys. Rev. B 69, 024511 (2004).

[55] P. Richard, G. Riou, I. Hetel, S. Jandl, M. Poirier, and P. Fournier, Phys. Rev. B 70, 064513 (2004).

[56] I. W. Sumarlin, J. W. Lynn, T. Chattopadhyay, S. N. Sarilo, D. I. Zhigunov, and J. L. Peng, Phys. Rev. B 51, 5824 (1995).

[57] We note that the calibration of the $\mathrm{Cu}^{2+}$ moment is based on normalizing magnetic scattering to the weak nuclear $(1,1,0)$ Bragg peak without considering absorption and extinction effects. In addition, since neutrons are a bulk probe and we can only estimate the moment by assuming an average ordered moment on all $\mathrm{Cu}$ sites. The errors only represent statistical uncertainties in the calculation.

[58] S. Skanthakumar, J. W. Lynn, J. L, Peng, and Z. Y. Li, J. Appl. Phys. 73, 6326 (1993).

[59] V. P. Plakhty, S. V. Maleyev, S. V. Gavrilov, F. Bourdarot, S. Pouget, and S. N. Barilo, Europhys. Lett. 61, 534 (2003).

[60] S. L. Li, S. D. Wilson, D. Mandrus, B. R. Zhao, Y. Onose, Y. Tokura, and Pengcheng Dai, Phys. Rev. B 71, 054505 (2005).

[61] Whether the 3D AF phase coexists with superconductivity macroscopically or microscopically in NCCO is still an open question. In addition, it is not clear why optimally doped NCCO has 3D AF order in coexistence with superconductivity while similar PLCCO does not.

[62] B. Khaykovich, S. Wakimoto, R. J. Birgeneau, M. A. Kastner, Y. S. Lee, P. Smeibidl, P. Vorderwisch, and K. Yamada, cond-mat/0411355 (unpublished). 